\title{
Twistor/ambitwistor strings and null-superstrings in spacetime of $D=4,10$ and 11 dimensions
}

\author{
Igor Bandos \\ Department of Theoretical Physics, University of the Basque Country, \\ UPV/EHU, P.O. Box 644, 48080 Bilbao, Spain \\ IKERBASQUE, Basque Foundation for Science, \\ 48013 Bilbao, Spain \\ E-mail: igor.bandos@ehu.es
}

ABSTRACT: We show that, at the classical level, the recently proposed 'ambitwistor string' model is equivalent to the spinor moving frame formulation of null-supersting, which in its turn is equivalent to Siegel's formulation of closed twistor string or to its higher dimensional generalizations. Although the null-(super)string is usually considered as describing the tensionless limit of (super)string, we show that its action can be derived from the spinor moving frame formulation of superstring also in the infinite tension limit. This observation, supplemented by some indirect arguments, allows us to conjecture the absence of critical dimensions, i.e. that the (ambi)twistor string based technique(s) to calculate field theory amplitudes can be developed not only in $\mathrm{D}=10$ or 26 , but also in $\mathrm{D}=11$ and other dimensions. The $\mathrm{D}=11$ and $\mathrm{D}=10$ twistor strings are described in some details.

Keywords: Superspaces, Superstrings and Heterotic Strings, Extended Supersymmetry, Scattering Amplitudes

ARXIV EPRINT: 1404.1299 


\section{Contents}

1 Introduction

2 Ambitwistor string action and kappa-symmetry of ambitwistor superstring

3 Ambitwistor string and null superstring. Moving frame enters the game

3.1 Moving frame formulation of null superstring as equivalent form of the ambitwistor string action

3.2 Null superstring action from tensionless and infinite tension limits of GreenSchwarz superstring

4 No critical dimensions for tensionless limit of superstring. Neither for the superstring of infinite tension.

5 Ambitwistor string as D-dimensional generalization of twistor (super)string. Spinor moving frame enters the game.

5.1 Spinor moving frame

5.2 Spinor moving frame action for null-superstring in $\mathrm{D}=4$ and Siegel's form of the twistor string action

5.3 Twistor string in $\mathrm{D}=11$ and $\mathrm{D}=10$ from ambitwistor/null superstring action 14 5.3.1 Spinor moving frame action for 11D ambitwistor superstring and its reformulation in the generalized 11D superspace 14

$\begin{array}{lll}\text { 5.3.2 } & 11 \mathrm{D} \text { twistor string action from ambitwistor superstring } & 16\end{array}$

$\begin{array}{lll}\text { 5.3.3 10D twistor string action from ambitwistor superstring } & 17\end{array}$

\section{Introduction}

In recent [1] Mason and Skinner proposed an 'ambitwistor' string and superstring models and argued that, upon quantization according the prescription discussed in [1], these contain only massless particles in the quantum state spectrum, are consistent in $D=10(D=26$ in the bosonic case) and reproduce the Cachazo-He-Yuan formulae for tree-level scattering amplitudes [2]. (In recent [3] these formulae for SYM amplitudes were proved using the BCFW (Britto-Cachazo-Feng-Witten) techique [4]). The NSR version of the ambitwistor string were also discussed in [1] and in [5], while the corresponding limit of the pure spinor formulation of superstring was the subject of [6].

According to [1] the ambitwistor string appears as an infinite tension limit of the standard Green-Schwarz (GS) superstring. On the other hand, the authors of [1] noticed the relation with the equations from the famous papers by Gross and Mende [7, 8], which described the string at ultra-high energy and due to this reason, usually associated with tensionless limit of string rather than with the limit of infinite tension. 
In this paper we show that, at the classical level, the ambitwistor string model is equivalent to null- superstring as described in moving frame and spinor moving frame formulation (see $[9-13]^{1}$ for $\mathrm{D}=4$ case). We also study the tensionless limit and the limit of infinite tension of the (spinor) moving frame formulation of the GS superstring, which was proposed in [23] and studied in $[24,25],{ }^{2}$ and show that, in suitable setups, both limits can produce the null-superstring action. This provides us with an explanation why the ambitwistor string, claimed to be the infinite tension limit of the superstring in [1], may reproduce the amplitudes with the properties characteristic for the tensionless limit of the superstring $[7,8]$.

Furthermore, it is known that the tensionless (limit of) string does not have critical dimensions (see [22, 36-38], refs in [22] and also [9-13]). This allows us to conjecture that the ambitwistor string also can be formulated and is consistent in an arbitrary dimension $D$, including in $\mathrm{D}=11$, where its quantization according (a generalization of) the scheme used in $[1,5,6]$ (or following the line of [39-41]) should produce (tree and one-loop) amplitudes for the 11 dimensional supergravity.

In [42] it was shown that the $\mathrm{D}=4 \mathcal{N}=4$ version of the null-supestring model [10-13] is equivalent to the closed twistor string model proposed by Siegel in [43] (see [44] for the original formulation, [45] for another open twistor string action, as well as [39-41, 46] and refs. in [41] for further development of the twistor string approach). In this paper we show that spinor moving frame formulation the D-dimensional null-superstring action, which is classically equivalent to the ambitwistor string, is also equivalent to the ' $D$-dimensional' generalization of the Siegel's twistor string action. Thus ambitwistor string can be also called twistor string.

In contrast with $\mathrm{D}=4$, the $\mathrm{D}=10,11$ versions of Siegel's twistor string, which we describe in some details, are formulated in terms of strongly constrained spinors related to the spinor moving frame variables of $[23-25,48,51,52]$. However, the similarity of nullsuperstring and superparticle action may simplify the quantization of such a constrained system. An interesting problem for future is to quantize the $D=10$ and $D=11$ twistor strings according to the line developed in $[51,52]$ for $11 \mathrm{D}$ superparticle, and to compare the results with [1] and with [6].

\section{Ambitwistor string action and kappa-symmetry of ambitwistor superstring}

The action for bosonic 'ambitwistor string' proposed by Mason and Skinner in [1] reads

$$
S_{\mathrm{MS}}^{\text {bosonic }}=\int_{\mathcal{W}^{2}} d^{2} \xi\left(P_{a} \bar{\partial} X^{a}-\frac{e}{2} P^{2}\right),
$$

where $\mathcal{W}^{2}$ is the two dimensional worldsheet with local coordinates $\xi^{m}=\left(\xi^{0}, \xi^{1}\right)$, $X^{a}=X^{a}(\xi)$ is a coordinate function describing the embedding of $\mathcal{W}^{2}$ as a surface in

\footnotetext{
${ }^{1}$ See [14-21] and refs. in [22] for other formulations of null-string and tensionless string action.

${ }^{2}$ See [26-35] for other approaches to superparticle and supertring models using the spinor moving frame variables (Lorentz harmonics)
} 
D-dimensional spacetime $M^{D}, a=0,1, \ldots,(D-1)$, and $P_{a}=P_{a}(\xi)$ and $e=e(\xi)$ are auxiliary fields. $\bar{\partial}$ is derivative in one of the directions of the worldsheet. If the signature is taken to be Euclidean, one can introduce a complex structure and related complex coordinates, $z=\xi^{0}+i \xi^{1}$ and $\bar{z}=\xi^{0}-i \xi^{1}$, and identify $\bar{\partial}=\partial_{\bar{z}}:=\frac{\partial}{\partial \bar{z}}$. Considering the case of Minkowski signature, one can use $\bar{\partial}=\partial_{=}=\frac{1}{2}\left(\partial_{0}-\partial_{1}\right)$. However, in this paper we will often use the notation $\bar{\partial}_{\bar{z}}=\bar{\partial}$ also for this case.

Eq. (2.1) differs from the massless particle action $S_{0}=\int_{W^{1}} d \tau\left(p_{a} \partial_{\tau} x^{a}-\frac{e}{2} p^{2}\right)$ only by replacing worldline $W^{1}$ by the worldsheet $\mathcal{W}^{2}$ and allowing all the field depend on two coordinates of the worldsheet.

The ambitwistor superstring action is obtained by substituting $\bar{z}$ component of the pull-back of the bosonic supervielbein form, $E_{\bar{z}}^{a}=\bar{\partial} Z^{M} E_{M}^{a}(Z)$ for $\bar{\partial} X^{a}$ in (2.2),

$$
S_{\mathrm{MS}}=\int_{\mathcal{W}^{2}} d^{2} \xi\left(P_{a} E_{\bar{z}}^{a}-\frac{e}{2} P^{2}\right) .
$$

In the case of flat target superspace $\Sigma^{(D \mid n)}$, which we are interested in, the supervielbein can be written in the form

$$
E^{a}=d X^{a}-i d \Theta \Gamma^{a} \Theta, \quad E^{\underline{\alpha}}=d \Theta^{\underline{\alpha}}
$$

where $\Theta^{\underline{\alpha}}$ are the fermionic coordinates of the superspace, and

$$
E_{\bar{z}}^{a}=\bar{\partial} X^{a}-i \bar{\partial} \Theta \Gamma^{a} \Theta, \quad E_{\bar{z}}^{\alpha}=\bar{\partial} \Theta^{\underline{\alpha}}
$$

appears in the decomposition of their pull-backs (which we denote by the same symbols)

$$
E^{a}=d \xi^{m} E_{m}^{a}=d z E_{z}^{a}+d \bar{z} E_{\bar{z}}^{a}, \quad E^{\underline{\alpha}}=d \Theta^{\underline{\alpha}}=d \xi^{m} \partial_{m} \Theta^{\underline{\alpha}}=d z \partial \Theta^{\underline{\alpha}}+d \bar{z} \bar{\partial} \Theta^{\underline{\alpha}} .
$$

These include $D$ bosonic and $n$ fermionic coordinate functions

$$
Z^{M}(\xi) \equiv Z^{M}(z, \bar{z})=\left(X^{a}(\xi), \Theta^{\underline{\alpha}}(\xi)\right), \quad a=0,1, \ldots,(D-1), \quad \underline{\alpha}=1, \ldots, n,
$$

where $n$ depends on $D$ and also on $\mathcal{N}$ in the case of $\mathcal{N}$-extended supersymmetry.

For $\mathrm{D}=4, \mathcal{N}$-extended superspaces (of which $\mathcal{N}=4$ case is relevant for the maximal $4 \mathrm{D}$ SYM theory), $a=0,1,2,3, n=4 \mathcal{N}, \Theta^{\underline{\alpha}}=\left(\theta_{i}^{\alpha}, \bar{\theta}^{\dot{\alpha} i}\right)$ with $\alpha=1,2, \dot{\alpha}=1,2, i=1, \ldots, \mathcal{N}$, and

$$
D=4: \quad \Gamma_{\underline{\alpha} \underline{\beta}}^{a}=\left(\begin{array}{cc}
0 & \sigma_{\alpha \dot{\beta}}^{a} \delta^{i}{ }_{j} \\
\sigma_{\beta \dot{\alpha}}^{a} \delta_{i}{ }^{j} & 0
\end{array}\right), \quad \text { with } \quad \alpha=1,2, \quad \dot{\alpha}=1,2, \quad i=1, \ldots, \mathcal{N},
$$

where $\sigma_{\beta \dot{\alpha}}^{a}=\epsilon_{\beta \alpha} \epsilon_{\dot{\alpha} \dot{\beta}} \tilde{\sigma}^{a \dot{\beta} \alpha}$ are relativistic Pauli matrices, $\sigma^{a} \tilde{\sigma}^{b}+\sigma^{b} \tilde{\sigma}^{a}=2 \eta^{a b} \mathbb{I}_{2 \times 2}$, so that

$$
E^{a}=d X^{a}-i d \theta_{i} \sigma^{a} \bar{\theta}^{i}+i d \theta_{i} \sigma^{a} \bar{\theta}^{i} .
$$

In the case of $\mathrm{D}=10, \mathcal{N}=1$ superspace, relevant for the $10 \mathrm{D}$ SYM theory, $a=0,1, \ldots, 9$, $\underline{\alpha}=1, \ldots, 16$ is the $10 \mathrm{D}$ Majorana-Weyl index, the indices of the $16 \times 16$ matrix $\Gamma_{\underline{\alpha} \underline{\beta}}^{a}=\sigma_{\underline{\alpha} \beta}^{a}=\sigma_{\underline{\beta} \underline{\alpha}}^{a}$ cannot be risen, but there exist $\tilde{\sigma}^{a \underline{\alpha} \underline{\beta}}=\tilde{\sigma}^{a \underline{\beta} \underline{\alpha}}=\tilde{\Gamma}^{a \underline{\alpha} \underline{\beta}}$ which obey $\sigma^{a} \overline{\tilde{\sigma}}^{b}+\sigma^{\bar{b}} \tilde{\sigma}^{a}=2 \eta^{a b} \mathbb{I}_{16 \times 16}$

$D=10: \quad \Gamma_{\underline{\alpha} \underline{\beta}}^{a}=\sigma_{\underline{\alpha} \underline{\beta}}^{a}, \quad \tilde{\Gamma}^{a \underline{\alpha} \underline{\beta}}=\tilde{\sigma}_{a}^{\underline{\alpha} \underline{\beta}}, \quad \underline{\alpha}, \underline{\beta}=1, \ldots, 16, \quad \sigma^{a} \tilde{\sigma}^{b}+\sigma^{b} \tilde{\sigma}^{a}=2 \eta^{a b} \mathbb{I}_{16 \times 16}$. 
Notice also the famous identity

$$
D=10: \quad \sigma_{a \underline{\alpha}(\underline{\beta}} \sigma_{\underline{\gamma} \underline{\delta})}^{a} \equiv 0
$$

which is very important in the Green-Schwarz (GS) superstring model. The classical GS superstring exists only in the dimensions $\mathrm{D}=3,4,6,10$ where a counterpart of (2.10) is valid. In contrast the existence of this identity is completely irrelevant for the ambitwistor superstring as defined by the action (2.2).

This is explained by close relation of (2.2) with the Brink-Schwarz superparticle action $S_{\mathrm{BS}}=\int_{W^{1}}\left(P_{a} E^{a}-d \tau \frac{e}{2} P^{2}\right)$ which basically consists in replacing worldline $W^{1}$ by the worldsheet $\mathcal{W}^{2}$ and allowing all the fields to depend on two worldsheet coordinates $\xi^{m}=(\tau, \sigma)$. Indeed, the local fermionic $\kappa$-symmetry, which leaves invariant the action $(2.2)$, is similar to the massless superparticle $\kappa$-symmetry [53]

$$
\delta_{\kappa} \Theta^{\underline{\alpha}}=P_{a} \tilde{\Gamma}^{a} \underline{\underline{\alpha}} \underline{\beta} \kappa_{\underline{\beta}}, \quad \delta_{\kappa} X^{a}=i \delta_{\kappa} \Theta \Gamma^{a} \Theta, \quad \delta_{\kappa} e=-2 i \Theta \kappa
$$

and relies only on the defining property of the $\Gamma$-matrix

$$
\left(\Gamma^{a} \tilde{\Gamma}^{b}+\Gamma^{b} \tilde{\Gamma}^{a}\right)_{\underline{\alpha}} \underline{\beta}=2 \eta^{a b} \delta_{\underline{\alpha}}^{\underline{\beta}} .
$$

This implies that, as in the case of massless superparticle [56], the classical 'ambitwistor string' of [1] , as described by the action (2.2), does exist in target superspace of any bosonic dimension. Below we will discuss an indication that this is true also for the quantum theory.

Thus we can consider also the $11 D$ ambitwistor superstring characterized by the action (2.2) with $a=0,1, \ldots, 9,10,(2.4)$ and

$$
D=11: \quad \Gamma_{\underline{\alpha} \underline{\beta}}^{a}=\Gamma_{\underline{\beta} \underline{\alpha}}^{a}=\left(C \tilde{\Gamma}^{a} C\right)_{\underline{\alpha} \underline{\beta}}, \quad C_{\underline{\alpha} \underline{\beta}}=-C_{\underline{\beta} \underline{\alpha}} \quad \underline{\alpha}, \underline{\beta}=1, \ldots, 32 .
$$

The quantization of this model along the (generalization of the) line of $[1,5]$ or $[6]$ presumably gives the formulae for tree-level amplitudes of 11D supergravity.

This statement is in contradiction with the point of view in $[1,5]$, where the stringy critical dimensions $\mathrm{D}=26$ and $\mathrm{D}=10$ are attributed to the bosonic and supersymmetric versions of ambitwistor string. Below we present some arguments in favor of that the ambitwistor string, being classically equivalent of null-superstring, does not have critical dimensions and can be defined in any $\mathrm{D}$ including $\mathrm{D}=11$.

To lighten the notation, from now on we will omit underlining of the Majorana and Majorana-Weyl spinor indices,

$$
\underline{\alpha}, \underline{\beta}, \underline{\gamma}, \ldots \quad \mapsto \quad \alpha, \beta, \gamma, \ldots,
$$

in all places where this cannot produce a confusion. We also will tend to use the shorter name ambitwistor string for the ambitwistor superstring. 


\section{Ambitwistor string and null superstring. Moving frame enters the game}

\subsection{Moving frame formulation of null superstring as equivalent form of the ambitwistor string action}

The Lagrange multiplier $e$ in (2.2) produces the mass shell conditions

$$
P_{a} P^{a}=0
$$

This is an algebraic equation so that its solution, if found, can be substituted into the action; thus one can write the action (2.2) as

$$
\begin{aligned}
S_{\mathrm{MS}}^{\prime} & =\left.\int_{\mathcal{W}^{2}} d^{2} \xi E_{\bar{z}}^{a} P_{a}\right|_{P^{2}=0} \\
& =\left.\int_{\mathcal{W}^{2}} d^{2} \xi P_{a}\right|_{P^{2}=0}\left(\bar{\partial} X^{a}+\text { fermions }\right) .
\end{aligned}
$$

At any point of $\mathcal{W}^{2}$ we can chose a suitable Lorentz frame to solve (3.1) by

$$
P_{(a)}=\rho(\xi)(1, \underbrace{0, \ldots, 0}_{D-2},-1)
$$

The general solution of (3.1) can be obtained from (3.3) by local (on $\mathcal{W}^{2}$ ) Lorentz rotation. This is to say it has the form

$$
P_{a}(\xi)=U_{a}^{(b)} P_{(b)}=\rho(\xi)\left(u_{a}^{0}-u_{a}^{(D-1)}\right)=: \rho^{\#}(\xi) u_{a}^{=}(\xi),
$$

where $U_{a}^{(b)}=\left(u_{a}^{0}, u_{a}^{1}, \ldots, u_{a}^{(D-1)}\right)$ is a Lorentz group valued matrix, called the moving frame matrix. We prefer to write it in terms of light-like vectors $u_{a}^{ \pm \pm}=u_{a}^{0} \pm u_{a}^{D-1}$ and to make notation more compact re-denoting $u_{a}^{--}=: u_{a}^{=}, u_{a}^{++}=: u_{a}^{\#}$,

$$
U_{a}^{(b)}(\xi)=\left(\frac{1}{2}\left(u_{a}^{=}+u_{a}^{\#}\right), u_{a}^{i}, \frac{1}{2}\left(u_{a}^{\#}-u_{a}^{=}\right)\right) \in \mathrm{SO}(1, D-1) .
$$

This matrix describes a local Lorentz frame adapted to an embedding of the worldsheet $\mathcal{W}^{2}$ into the spacetime in such a way that the spacial component of the momentum density at point $\xi, P_{a}(\xi)$, is oriented along the (D-1)-th axis and has a negative projection on this axis.

The fact that the moving frame matrix $U$ belongs to $O(1, D-1)$ is expressed by the constraint $U^{T} \eta U=\eta$, which implies that its columns are orthogonal and normalized; in terms of $u_{a}^{=}(\xi)=u_{a}^{0}-u_{a}^{(D-1)}$ and $u_{a}^{\#}(\xi)=u_{a}^{0}+u_{a}^{(D-1)}$ these properties are expressed by

$$
\begin{array}{rlrl}
u_{a}^{=} u^{a=} & =0, & \\
u_{a}^{\#} u^{a \#} & =0, & & u_{a}^{=} u^{a \#}=2, \\
u_{a}^{i} u^{a=} & =0=u_{a}^{i} u^{a \#}, & u_{a}^{i} u^{a j}=-\delta^{i j} .
\end{array}
$$


Equivalently, the statement of $(3.5), U_{a}^{(b)}(\xi) \in O(1, D-1)$, can be expressed by 'unity decomposition' $U \eta U^{T}=\eta$, i.e.

$$
\delta_{a}{ }^{b}=\frac{1}{2} u_{a}^{=} u^{b \#}+\frac{1}{2} u_{a}^{\#} u^{b=}-u_{a}^{i} u^{b i} .
$$

The fact that $U$ belongs to $\mathrm{SO}(1, D-1)$ subgroup of $O(1, D-1)$ implies that $\operatorname{det} U_{a}^{(b)}=1$ i.e. that

$$
\begin{aligned}
& \epsilon^{a b c_{1} \ldots c_{D-2}} u_{b}^{\bar{E}} u_{c_{1}}^{i_{1}} \ldots u_{c_{D-2}}^{i_{D-2}}=-(-)^{D} u^{a=} \epsilon^{i_{1} \ldots i_{D-2}}, \\
& \epsilon^{a b c_{1} \ldots c_{D-2}} u_{b}^{\#} u_{c_{1}}^{i_{1}} \ldots u_{c_{D-2}}^{i_{D-2}}=+(-)^{D} u^{a \#} \epsilon^{i_{1} \ldots i_{D-2}} .
\end{aligned}
$$

The splitting (3.5) of the moving frame matrix is invariant under local $\mathrm{SO}(1,1) \otimes$ $\mathrm{SO}(D-2)$ transformations; $u_{a}^{i}$ transforms as $\mathrm{SO}(D-2)$ vector, while the light-like vectors $u_{a}^{=}$and $u_{a}^{\#}$ carry the weights -2 and +2 of $\mathrm{SO}(1,1)$ group,

$$
u_{a}^{=} \mapsto u_{a}^{=} \exp \{-2 \beta\}, \quad u_{a}^{\#} \mapsto u_{a}^{\#} \exp \{2 \beta\}, \quad u_{a}^{i} \mapsto u_{a}^{j} \mathcal{O}^{j i}, \quad \mathcal{O O}^{T}=\mathbb{I}_{(D-2) \times(D-2)} .
$$

To make invariant the solution (3.4) of the constraint (3.1), we have to assume that $\rho$ in $(3.4)$ carries $\mathrm{SO}(1,1)$ weight +2 , which is reflected by denoting $\rho=\rho^{\#}$ in the last part of eq. (3.4),

$$
\rho^{\#} \mapsto \rho^{\#} \exp \{2 \beta\}
$$

Substituting the general solution (3.3) of the constraint (3.1) into (2.2) (or (3.2)) we arrive at following moving frame action for ambitwistor string

$$
S_{\mathrm{MF}}=\int_{\mathcal{W}^{2}} d^{2} \xi \rho^{\#} E_{\bar{z}}^{=}:=\int_{\mathcal{W}^{2}} d^{2} \xi \rho^{\#} E_{\bar{z}}^{a} u_{a}^{=} .
$$

One can also write this action as an integral of differential form:

$$
S_{\mathrm{MF}}=\frac{i}{2} \int_{\mathcal{W}^{2}} d z \wedge \rho^{\#} E^{=}:=\frac{i}{2} \int_{\mathcal{W}^{2}} d z \wedge d \bar{z} E_{\bar{z}}^{a} u_{a}^{=} \rho^{\#} .
$$

Let us compare the above equivalent form of the ambitwistor string action with (D-dimensional generalization of the) null-superstring action of [10-13], which can be written as

$$
S_{\text {null }}=\int_{\mathcal{W}^{2}} d^{2} \xi \rho^{\# m} E_{m}^{=}:=\int_{\mathcal{W}^{2}} d^{2} \xi \rho^{\# m} E_{m}^{a} u_{a}^{=},
$$

or, in terms of differential forms, as

$$
S_{\text {null }}=\int_{\mathcal{W}^{2}} \tilde{e}^{\#} \wedge E^{=}:=\int_{\mathcal{W}^{2}} d^{2} \xi \tilde{e}^{\#} \wedge E^{a} u_{a}^{=},
$$

where $\tilde{e}^{\#}=d \xi^{m} \tilde{e}_{m}^{\#}(\xi)$ and $\tilde{e}_{m}^{\#}(\xi) \propto \epsilon_{m n} \rho^{n}$.

The equivalent form (3.13) (and (3.14)) of the ambitwistor string action can be considered as a gauge fixed version of the null-superstring action (3.15) (and (3.16)) in which the $2 \mathrm{~d}$ diffeomorphism gauge symmetry is broken by the condition

$$
\rho^{\# m}=\rho^{\#} \delta_{\bar{z}}^{m}
$$

the residual part of the diffeomorphism gauge symmetry of the action (3.13) (and (3.14)) which preserves (3.17), can be identified as $2 \mathrm{~d}$ conformal symmetry transformations, $z \mapsto \bar{f}(z), \bar{\partial} f(z)=0$. 


\subsection{Null superstring action from tensionless and infinite tension limits of Green-Schwarz superstring}

Now we would like to address the question of what is the limit in which the null-superstring can be obtained from GS superstring. Let us start with (spinor)moving frame formulation of the GS supersting action [23-25]

$$
S_{\mathrm{GS}}=T \int_{\mathcal{W}^{2}}\left(e^{\#} \wedge E^{=}+e^{=} \wedge E^{\#}-e^{\#} \wedge e^{=}\right)-T \int_{\mathcal{W}^{2}} B_{2} .
$$

In it (cf. (3.13), (3.14))

$$
E^{=}=E^{a} u_{a}^{=}, \quad E^{\#}=E^{a} u_{a}^{\#}
$$

are projections of the pull-back of the target space bosonic supervielbein on the light-like vector fields of the moving frame (3.5), $e^{\#}=d \xi^{m} e_{m}^{\#}(\xi)$ and $e^{=}=d \xi^{m} e_{m}^{=}(\xi)$ are independent worldvolume 1-forms on $\mathcal{W}^{2}, \int_{\mathcal{W}^{2}} B_{2}$ is the Wess-Zumino term; its explicit form, which is the same as in the standard formulation of the GS superstring, will not be needed here. Finally,

$$
T=\frac{1}{4 \pi \alpha^{\prime}}
$$

is the superstring tension and $\alpha^{\prime}$ is the Regge slop parameter. An equivalent form of the (spinor)moving frame action is

$$
S_{\mathrm{GS}}=T \int_{\mathcal{W}^{2}} d^{2} \xi\left(\rho^{\# m} E_{m}^{=}+\rho^{=m} E_{m}^{\#}-\epsilon_{m n} \rho^{\# m} \rho^{=m}\right)-T \int_{\mathcal{W}^{2}} B_{2},
$$

where $\rho^{\# m} \propto \epsilon^{m n} e_{n}^{\#}$ and $\rho^{=m} \propto \epsilon^{m n} e_{n}^{=}$.

Clearly, if we set $T=0$ without redefining the basic variables, the action just vanishes. However, if we first set $e^{\#}=\tilde{e}^{\#} / T=4 \pi \alpha^{\prime} \tilde{e}^{\#}$ and then take the $T \mapsto 0$ limit keeping $\tilde{e}^{\#}$ 'fixed', the action (3.18) reduces to (3.13),

$$
\lim _{T \mapsto 0}\left[\left.S_{\mathrm{GSS}}\right|_{e^{\#}=\tilde{e} \# / T}\right]=S_{\text {null }} .
$$

This is the reason to consider the null-superstring as tensionless limit of the GS superstring (and the bosonic null-string as tensionless limit of the bosonic string).

To relate the null superstring, and hence ambitwistor superstring action, to the infinite tension limit of the above action for GS superstring, we need to perform the redefinition with opposite rescaling $e^{\#}=T \tilde{e}^{\#}$ (or $\rho^{\# m} \mapsto T \rho^{\# m}$ ). Then we find

$$
\left.S_{\mathrm{GS}}\right|_{e^{\#}=T \tilde{e}^{\#}}=T^{2}\left[S_{\text {null }}+\mathcal{O}\left(\frac{1}{T}\right)\right] \text {, }
$$

which is dominated by contribution of null superstring action in the limit of infinite tension,

$$
\lim _{T \mapsto \infty}\left[\frac{\left.S_{\mathrm{GS}}\right|_{e^{\#}=T \tilde{e}^{\#}}}{T^{2}}\right]=S_{\text {null }} .
$$

To reproduce the wanted ambitwistor string in this limit we renormalized the action thus making its dimension different from $[\hbar](=1$ in our conventions). This is not a problem 
for classical theory, as far as in it the action is used only to derive the equations of motion through the variational principle. In quantum theory this argument does not work as far as the action should be exponentiated. This implies that we need to renormalize the action or our basic variable, $\tilde{e}^{\#}$ or $\rho^{\#}$, using another dimensionfull constant.

Notice that such a constant should certainly be present in the ambitwistor string approach of [1] for calculating scattering amplitudes. Indeed, the proposition of $[1,5]$ is to calculate the amplitudes for 10D SYM, which is not a conformally invariant theory and has a dimensionfull coupling constant $\left(\left[g_{10 D}^{Y M}\right]=L^{-3}\right) .{ }^{3}$ It would be interesting to follow in some details the appearance of the coupling constant in the approach of $[1,5,6]$, but this is certainly out of the score of the present paper. ${ }^{4}$ Here we just consider the above discussion as a suggestion that, upon a suitable use of an additional dimensional constant (which is certainly present in the models of interest for the ambitwistor string program) the infinite tension limit of classical GS superstring can also be described by the null-superstring action.

This does not mean that infinite tension limit of superstring is identical to the tensionless limit in quantum theory. Although the classical actions coincide, the ambitwistor string approach [1] uses the vertex operators which may be different from the ones of the tensionless string; in particular, as it was stressed in [1], despite the close similarity with the equations from Gross and Mende articles [7, 8], the amplitudes in tensionless and infinite tension limits are actually different.

In the next section we present indirect arguments in favor of one more similarity between ambitwistor string and tensionless superstring: namely of that in both limits the model can be formulated in spacetime of arbitrary dimensions.

\footnotetext{
${ }^{3}$ This prescription does not work, at least in its literal form, in $\mathrm{D}=4$ case where the SYM coupling constant $g_{4 D}^{Y M}$ is dimensionless, $\left[g_{4 D}^{Y M}\right]=1$. Curiously, this is the case where it had been established that the twistor string produces the tree amplitudes for $\mathcal{N}=4 \mathrm{SYM}[44,45]$. This fact is relevant for our study because, as we show in section 5 , the twistor string is equivalent to the ambitwistor string at the classical level. On the other hand, studying the loops of the $\mathrm{D}=4$ twistor string, Berkovits and Witten found [79] that these give rise to the amplitudes of conformal rather than Einstein supergravity. As this is not a consequent theory, the problem of modifying the model in such a way that it's gravity sector is Einstein arose and was addressed in [80] (see also [41] and refs therein). Such a modification inevitably involves a dimensional constant $\kappa$ of Einstein gravity so that, in the light of the classical equivalence of twistor string and $\mathrm{D}=4$ null-superstring [42], it is tempting to speculate that just $\kappa$ have to be used to correct the dimension of the variables appearing in the term dominating the infinite tension limit of the superstring action in such a way that it becomes the dimensionless $([S]=[\hbar])$ null-superstring action.

${ }^{4}$ Let us also stress that ambitwistor string action as proposed in [1], eq. (2.1), does possess the conformal invariance which is not the case for the SYM models in $D \neq 4$. This reflects the fact that, besides the action, some additional tools are needed to reproduce the correlation functions and scattering amplitudes. This is similar to the situation with recently proposed action $[57,58]$ for the interacting 'higher spin gravity' theories: it reproduces the 'unfolded' equations by Vasiliev [59-61] but, after linearization, does not reproduce the propagators of Fronsdal's theory [62].

One can also notice a similarity with Berkovits pure spinor approach to quantum superstring [63], which uses just a free conformal field theory action for the standard superstring variables, pure spinors and their momentum, while the actual content of the theory, its identification with superstring, is fixed by postulating the BRST charge; also a prescription for path integral measure and its regularization were necessary [64, 65] and are still under active study [66]. Notice however the absence of fundamental $b$ and $c$ ghosts in the pure spinor approach: the $b$ ghost is composite and its properties were the subject of separate study [67].
} 


\section{No critical dimensions for tensionless limit of superstring. Neither for the superstring of infinite tension.}

It is important that the quantum theory of tensionless (super)string exists for any spacetime dimensions $\mathrm{D}[10-13,22,37,38]$. In the critical dimensions, where the quantum (super)string with finite tension exists, this can be understood studying the tensionless limit of quantum superstring, $\alpha^{\prime} \mapsto \infty[22,37,38]$. To this end one observes that, after a proper normalization of the physical operators of string theory (with dimensionless oscillators $a_{n}^{a}, a_{-n a}=a_{n a}^{\dagger}$ obeying $\left[a_{n a}, a_{m b}^{\dagger}\right]=\delta_{n, m} \eta_{a b}$ ), the Virasoro generators read (see e.g. $\left.[38] ; a_{n}^{\dagger} \cdot a_{n}:=a_{n b}^{\dagger} a_{n}^{b}\right)$

$$
\begin{aligned}
L_{0} & =\frac{\alpha^{\prime}}{2} p_{a} p^{a}+\sum_{n>0} n a_{n}^{\dagger} \cdot a_{n} \\
L_{n>0} & =-i \sqrt{2 n \alpha^{\prime}} p_{a} a_{n}^{a}+\sum_{m>0} \sqrt{m(m+n)} a_{m}^{\dagger} \cdot a_{m+n}-\frac{1}{2} \sum_{m=1}^{n-1} \sqrt{m(m+n)} a_{m} \cdot a_{n-m}=\left(L_{-n}\right)^{\dagger} .
\end{aligned}
$$

Notice that, at small tension $T$, this is to say at large $\alpha^{\prime}$ (3.20), the oscillator terms are clearly sub-leading. Then the true symmetry generators in the tensionless limit $\alpha^{\prime} \mapsto \infty$ are defined by renormalized operators

$$
\begin{aligned}
L_{0} / \alpha^{\prime} \underset{\alpha^{\prime} \rightarrow \infty}{\longmapsto} & l_{0}=\frac{1}{2} p_{a} p^{a}, \\
L_{n} / \sqrt{\alpha^{\prime}} \underset{\alpha^{\prime} \rightarrow \infty}{\longmapsto} & l_{n}=-i \sqrt{2 n} p_{a} a_{n}^{a},
\end{aligned}
$$

which obeys the Heisenberg algebra with central element $l_{0}$,

$$
\left[l_{n}, l_{m}\right]=\delta_{n+m} l_{0}, \quad\left[l_{n}, l_{0}\right]=0, \quad n \neq 0,
$$

rather than Virasoro algebra. ${ }^{5}$

Hence tensionless superstring can be defined in spacetime with arbitrary dimension $D$ using string-inspired creation and annihilation operators. With these variables the spacetime conformal invariance is provided by that the quantum state spectrum contains only massless D-dimensional particles, which form massless supermultiplets in the case of supersymmetric tensionless string.

Now let us discuss the opposite, infinite tension limit $T=\frac{1}{4 \pi \alpha^{\prime}} \mapsto \infty$ which corresponds to $\alpha^{\prime} \mapsto 0$. The naïve limit of the original Virasoro constraints is clearly dominated by the oscillator terms. But this contradicts the intuitive expectation that at infinite tension the string looks like particle, so that rather the center of energy motion should be more important. This suggests to redefine the momentum variable as

$$
p_{a} \mapsto \tilde{p}_{a}=\alpha^{\prime} p_{a},
$$

\footnotetext{
${ }^{5}$ The critical dimensions are absent also when one quantizes the tensionless string in terms of coordinate functions and conjugate momentum variables [9-13], however in this case the spectrum of mass of the theory is continuous.
} 
write the Virasoro constraints in this terms, and renormalize them by suitable positive powers of $\alpha^{\prime}$ before taking the $\alpha^{\prime} \mapsto 0(T \mapsto \infty)$ limit. In such a way we again reproduce the Heisenberg algebra operators (4.5)

$$
\begin{aligned}
& \alpha^{\prime} L_{0} \underset{\alpha^{\prime} \rightarrow 0}{\longrightarrow} \quad l_{0}=\frac{1}{2} \tilde{p}_{a} \tilde{p}^{a} ， \\
& L_{n} / \sqrt{\alpha^{\prime}} \underset{\alpha^{\prime} \rightarrow 0}{\longrightarrow} \quad l_{n}=-i \sqrt{2 n} \tilde{p}_{a} a_{n}^{a},
\end{aligned}
$$

but now written in terms of renormalized $\tilde{p}_{a}(4.6)$.

Certainly the above discussion is not given in terms of variables of the spinor moving frame formulation of null-superstring and has a character of suggestion rather than of the proof; the quantum theory of null-superstring is beyond the scope of this paper. Notice, however, that the rescaling (4.6) $p_{a} \mapsto \tilde{p}_{a}=\alpha^{\prime} p_{a}$ is in correspondence with $e^{\#}=T \tilde{e}^{\#}$ (or $\left.\rho^{\#} \mapsto T \rho^{\#}\right)$ which was needed to reproduce the $\mathrm{D}=3,4,6,10$ null superstring action as a limit of GS superstring action in the corresponding dimension.

The above observations suggest that null-superstring, which, as we have shown in section 3.1, gives one of the classically equivalent formulations of the ambitwistor string of [1], exists in any spacetime dimensions, ${ }^{6}$ including say $D=11$, where the tensionfull superstring (superstring with nonzero tension) does not exist. Development of a generalization of technique of $[1,5,6]$ for this case might provide us with a tool to calculate tree and one-loop amplitudes of 11D supergravity.

\section{Ambitwistor string as D-dimensional generalization of twistor (super)string. Spinor moving frame enters the game.}

As it was shown in [42], the $\mathrm{D}=4 \mathcal{N}=4$ version of the twistor-like formulation of nullsuperstring [10-13] is equivalent to the closed twistor string action proposed by Siegel in [43] (see [44] for original formulation and [45] for an alternative action for twistor string). Actually this gives one more indirect argument in favor of our conjecture on the absence of critical dimension for the infinite tension limit of superstring, as on one hand, according to section 3, it can be described using the null-superstring action, and, on the other hand, twistor string is known to give a consistent (anomaly free) theory in $\mathrm{D}=4$. But this is not the end of story.

It is natural to expect that D-dimensional null-superstring, and hence ambitwistor string, also gives rise to a D-dimensional twistor superstring. In this section we show that this is indeed the case for $\mathrm{D}=11, \mathrm{D}=10$ and $4 \mathrm{D} \mathcal{N}$-extended null-superstring.

\footnotetext{
${ }^{6}$ One may think about a possibility to rescale also the central charges so that they remain in the tensionless limit. If realized, such a prescription would be clearly equivalent to taking the limit without any rescaling. In this respect we have to stress that the statement on absence of critical dimensions implies an existence of a possibility to chose the set of basic variables in such a way that the quantum theory can be formulated in terms of these and is consistent in a spacetime of arbitrary dimensions (rather than nonexistence of such variables that quantization in terms of these result in D-dependent anomaly). An example is the quantization of bosonic null string in terms of original stringy oscillators [17] which reproduces the stringy result on critical dimensions $\mathrm{D}=26$ also in the tensionless superstring limit. See [9-13] for more discussion on this issue.
} 
The spinor moving frame formulation of null-superstrings is related to corresponding formulation of massless superparticle in the same manner as originally proposed ambitwistor string action is related to the Brink-Schwarz superparticle action: basically by replacing all the functions of proper time $\tau$ by the functions of two worldsheet coordinates, $\xi^{m}=(z, \bar{z})$. Thus the D-dimensional null-superstring actions can be easily written starting form the spinor moving frame formulations of the massless superparticle actions presented in [47] for $\mathrm{D}=4$, in [48] for $\mathrm{D}=3,4,6$ and 10 and in [49-52] for $\mathrm{D}=11$.

\subsection{Spinor moving frame}

The moving frame formulation of the null-superstring (3.15) can be considered as twistorlike spinor moving frame formulation in the following sense.

For every $\mathrm{SO}(1, D-1)$ valued matrix $U$, including for the moving frame matrix (3.5), one can define the matrix $V \in \operatorname{Spin}(1, D-1)$ doubly covering $U$ in the sense of that

$$
V \Gamma_{b} V^{T}=U_{b}^{(a)} \Gamma_{(a)}, \quad(a) \quad V^{T} \tilde{\Gamma}^{(a)} V=\tilde{\Gamma}^{b} u_{b}^{(a)}, \quad(b) \quad V C V^{T}=C, \quad(c) .
$$

These equations reflect the Lorentz invariance of the Dirac (or Pauli) matrices $\Gamma^{a}$ and of the charge conjugation matrix (when this exists in the minimal spinor representation)

The $\mathrm{SO}(1,1) \otimes \mathrm{SO}(D-2)$ invariant splitting of the moving frame matrix $U$ is reflected by a splitting of its doubly covering spinor moving frame matrix $V$ on two rectangular blocks carrying different $\mathrm{SO}(1,1)$ weights and either different or the same representations of $\operatorname{Spin}(D-2)$ group. For the 11D case the spinor moving frame matrix reads

$$
D=11: \quad V_{(\beta)}^{\alpha}=\left(\begin{array}{c}
v_{q}^{+\alpha} \\
v_{q}^{-\alpha}
\end{array}\right) \in \operatorname{Spin}(1,10), \quad \alpha=1, \ldots, 32, \quad q=1, \ldots, 16 .
$$

There $16 \times 32$ blocks, $v_{q}^{-\alpha}$ and $v_{q}^{+\alpha}$, called spinor moving frame variables, carry the opposite $\mathrm{SO}(1,1)$ weights and the same 16 dimensional real (Majorana) spinor representation of $\mathrm{SO}(9)$.

In $\mathrm{D}=10$, the $16 \times 16$ spinor moving frame matrix $V$

$$
D=10: \quad V_{(\beta)}^{\alpha}=\left(\begin{array}{c}
v_{\dot{q}}^{+\alpha} \\
v_{q}^{-\alpha}
\end{array}\right) \in \operatorname{Spin}(1,9), \quad \alpha=1, \ldots, 16, \quad\left\{\begin{array}{l}
\dot{q}=1, \ldots, 8 \\
q=1, \ldots, 8
\end{array}\right.
$$

is split on $8 \times 16$ blocks, $v_{\dot{q}}^{+\alpha}$ and $v_{q}^{-\alpha}$, carrying $c-$ and $s-$ spinorial representations of $\mathrm{SO}(8)$.

In both $\mathrm{D}=11$ and $\mathrm{D}=10$ cases, the moving frame variables are strongly constrained by (5.1). In particular, the lower diagonal block of $(5.1 \mathrm{a})$ and ${ }^{(a)}={ }^{=}$component of $(5.1 \mathrm{~b})$ give the following constraints involving the light-like vector $u_{a}^{=}$,

$$
D=10,11: \quad v_{q}^{-\alpha}\left(\Gamma^{a}\right)_{\alpha \beta} v_{p}^{-\beta}=\delta_{q p} u_{a}^{=}, \quad 2 v_{q}^{-\alpha} v_{q}^{-\beta}=u_{a}^{=} \tilde{\Gamma}^{a \alpha \beta} .
$$

Actually, these are constraints for the spinor moving frame variables $v_{q}^{-\alpha}$, while the vector $u_{a}^{=}$is defined by these constraints and its property to be light-like is determined by them. The remaining blocks of the matrix constraint (5.1a) and components of (5.1b) involve the second spinor moving frame variable $v_{\dot{q}}^{+\alpha}(\dot{q}$ can be identified with $q$ in $\mathrm{D}=11$ case), define 
the other moving frame vectors, $u_{a}^{\#}$ and $u_{a}^{i}$, and determine their properties listed in (3.7). To resume, (with a suitable gamma matrix representation) the constraints $(5.1 \mathrm{a}, \mathrm{b})$ imply

$$
\begin{aligned}
v_{q}^{-} \Gamma^{a} v_{p}^{-} & =u_{a}^{=} \delta_{q p}, & 2 v_{q}^{-\alpha} v_{q}^{-\beta} & =u_{a}^{=} \tilde{\Gamma}^{a \alpha \beta}, \\
v_{\dot{q}}^{+} \Gamma_{a} v_{\dot{p}}^{+} & =u_{a}^{\#} \delta_{\dot{q} \dot{p},}, & 2 v_{\dot{q}}^{+\alpha} v_{\dot{q}}^{+\beta} & =\tilde{\Gamma}^{a \alpha \beta} u_{a}^{\#}, \\
v_{q}^{-} \Gamma_{a} v_{\dot{p}}^{+} & =-u_{a}^{i} \gamma_{q \dot{p}}^{i}, & 2 v_{q}^{-(\alpha} \gamma_{q \dot{q}}^{i} v_{\dot{q}}^{+\beta} & =-\tilde{\Gamma}^{a \alpha \beta} u_{a}^{i} .
\end{aligned}
$$

Here, for $\mathrm{D}=10 \gamma_{q \dot{p}}^{i}=\tilde{\gamma}_{\dot{p} q}^{i}$ are the $\mathrm{d}=8$ gamma (actually sigma) matrices, obeying $\gamma^{i} \tilde{\gamma}^{j}+$ $\gamma^{j} \tilde{\gamma}^{i}=\delta^{i j} I_{8 \times 8}$ (see e.g. [23-25, 48] for their properties), while for $\mathrm{D}=11 \dot{q}, \dot{p}$ must be identified with $q, p$ and the $16 \times 16$ Dirac matrices $\gamma_{q \dot{p}}^{i} \equiv \gamma_{q p}^{i}$ are real, symmetric $\gamma_{q p}^{i}=\gamma_{p q}^{i}$ and obey $\gamma^{i} \gamma^{j}+\gamma^{j} \gamma^{i}=2 \delta^{i j} I_{16 \times 16}$ (see [78] for more details). The constraint (5.1c) allows to express the elements of the inverse $11 \mathrm{D}$ spinor moving frame matrix through the same $v_{q}^{ \pm \beta}$,

$$
D=11: \quad v_{\alpha_{q}}^{-}=i C_{\alpha \beta} v_{q}^{-\beta}, \quad v_{\alpha q}^{+}=-i C_{\alpha \beta} v_{q}^{+\beta} .
$$

In $\mathrm{D}=10$ this constraint is absent (as far as the charge conjugartion matrix does not exists in the $\mathrm{D}=10$ Majorana-Weyl spinor representation) and the elements of the inverse spinor moving frame matrix, $v_{\alpha}^{+q}$ and $v_{\alpha}^{-\dot{q}}$, should be introduced as additional variables

$$
D=10: \quad V_{\alpha}^{(\beta)}=\left(v_{\alpha}^{+q}, v_{\alpha}^{-\dot{q}}\right) \in \operatorname{Spin}(1,9), \quad \alpha=1, \ldots, 16, \quad\left\{\begin{array}{l}
\dot{q}=1, \ldots, 8 \\
q=1, \ldots, 8
\end{array}\right.
$$

constrained by $V_{\alpha}^{(\beta)} V_{(\beta)}^{\gamma}:=v_{\alpha}^{-\dot{q}} v_{\dot{q}}^{+\gamma}+v_{q}^{-\alpha} v_{q}^{-\gamma}=\delta_{\alpha}^{\gamma}$ and

$$
\begin{array}{ll}
v_{q}^{-\alpha} v_{\alpha}^{+p}=\delta_{q p}, & v_{q}^{-\alpha} v_{\alpha}^{-\dot{q}}=0, \\
v_{\dot{q}}^{+\alpha} v_{\alpha}^{+p}=0, & v_{\dot{q}}^{+\alpha} v_{\alpha}^{-\dot{p}}=\delta_{\dot{q} \dot{p} .} .
\end{array}
$$

In terms of the elements of inverse spinor moving frame matrix the constraints (5.5)-(5.7) read

$$
\begin{aligned}
v_{q}^{+} \tilde{\Gamma}^{a} v_{p}^{+} & =u_{a}^{\#} \delta_{q p}, & 2 v_{\alpha}^{+q} v_{-\beta}^{+q} & =u_{a}^{\#} \Gamma_{\alpha \beta}^{a}, \\
v_{\dot{q}}^{-} \tilde{\Gamma}_{a} v_{\dot{p}}^{-} & =u_{a}^{=} \delta_{\dot{q} \dot{p},}, & 2 v_{\alpha}^{-\dot{q}} v_{\beta}^{-\dot{q}} & =\Gamma_{\alpha \beta}^{a} u_{a}^{\#}, \\
v_{q}^{+} \tilde{\Gamma}_{a} v_{\dot{p}}^{-} & =u_{a}^{i} \gamma_{q \dot{p}}^{i}, & 2 v_{(\alpha \mid}^{+q} \gamma_{q \dot{q}}^{i} v_{\mid \beta)}^{-\dot{q}} & =\Gamma_{\alpha \beta}^{a} u_{a}^{i} .
\end{aligned}
$$

In the case of $\mathrm{D}=4, \operatorname{Spin}(1,3)=\mathrm{SL}(2, \mathbb{C})$ so that the spinor moving frame can be defined by complex unimodular $2 \times 2$ matrix,

$$
D=4: \quad V_{(\beta)}^{\alpha}=\left(V_{(\dot{\beta})}^{\dot{\alpha}}\right)^{*}=\left(\begin{array}{c}
v^{+\alpha} \\
v^{-\alpha}
\end{array}\right) \in \mathrm{SL}(2, \mathbb{C}), \quad \alpha=1,2, \quad \dot{\alpha}=1,2
$$

so that $v_{\alpha}^{-}$can be considered as nonvanishing, but in all other respect unconstrained complex Weyl spinor, while $v_{\alpha}^{+}$is restricted by the normalization condition $v^{-\alpha} v_{\alpha}^{+}=1$ only, ${ }^{7}$

$$
\begin{array}{rlll}
D=4: & \operatorname{det}\left(V_{(\beta)}^{\alpha}\right)=1 & \Leftrightarrow & v^{-\alpha} v_{\alpha}^{+}=1 \\
\operatorname{det}\left(V_{(\dot{\beta})}^{\dot{\alpha}}\right)=1 & \Leftrightarrow & v^{-\dot{\alpha}} v_{\dot{\alpha}}^{+}=1 .
\end{array}
$$

\footnotetext{
${ }^{7}$ In our notation $\epsilon^{12}=-\epsilon_{12}=1$ so that $\operatorname{det} V=-\epsilon_{\alpha \beta} v^{+\alpha} v^{-\beta}=v^{-\alpha} v_{\alpha}^{+}$.
} 
This can be put in correspondence with (5.1c), while eqs. (5.1a,b) just define the set of light-like moving frame vectors as direct products of $v_{\alpha}^{ \pm}$and $\bar{v}_{\dot{\alpha}}^{ \pm}=\left(v_{\alpha}^{ \pm}\right)^{*},{ }^{8}$

$$
\begin{array}{ll}
D=4: \quad u_{a}^{ \pm \pm}=v^{ \pm} \sigma_{a} \bar{v}^{ \pm}=\left(u_{a}^{ \pm \pm}\right)^{*} \quad \Leftrightarrow \quad u_{a}^{ \pm \pm} \sigma_{\alpha \dot{\alpha}}^{a}=2 v_{\alpha}^{ \pm} \bar{v}_{\dot{\alpha}}^{ \pm} \\
u_{a}^{-+}=v^{-} \sigma_{a} \bar{v}^{+}=\left(u_{a}^{+-}\right)^{*} \quad \Leftrightarrow \quad u_{a}^{ \pm \mp} \sigma_{\alpha \dot{\alpha}}^{a}=2 v_{\alpha}^{ \pm} \bar{v}_{\dot{\alpha}}^{\mp} .
\end{array}
$$

\subsection{Spinor moving frame action for null-superstring in $\mathrm{D}=4$ and Siegel's form of the twistor string action}

We denote the coordinates of $\mathcal{N}$-extended $\mathrm{D}=4$ superspace by $\left(X^{a}, \theta_{i}^{\alpha}, \bar{\theta}^{\dot{\alpha} i}\right)$ with $a=0,1,2,3, \alpha=1,2, \dot{\alpha}=1,2$ and $i=1, \ldots, \mathcal{N}$. The action for the spinor moving frame formulation of null-superstring in this superspace can be obtained from the corresponding moving frame action, which in its gauge fixed version has the form (3.13), (3.14), by using $u_{a}^{=}=v^{-} \sigma_{a} \bar{v}^{-}$,

$$
S_{\mathrm{smf}}^{4 D}=\int_{\mathcal{W}^{2}} d^{2} \xi \rho^{\#} E_{\bar{z}}^{a} u_{a}^{=}=\int_{\mathcal{W}^{2}} d^{2} \xi \rho^{\#} v_{\alpha}^{-} \bar{v}_{\dot{\alpha}}^{-}\left(\bar{\partial} X^{a} \tilde{\sigma}_{a}^{\dot{\alpha} \alpha}-2 i \bar{\partial} \theta_{i}^{\alpha} \bar{\theta}^{\dot{\alpha} i}+2 i \theta_{i}^{\alpha} \bar{\partial} \bar{\theta}^{\dot{\alpha} i}\right) .
$$

It is similar to the superparticle action considered in [69] which is known to allow for change of variables to Ferber supertwistor [68]

$$
\mathcal{Z}_{\Lambda}=\left(\Upsilon_{\underline{\alpha}} ; \eta_{i}\right)=\left(\lambda_{\alpha}, \mu^{\dot{\alpha}} ; \eta_{i}\right)
$$

and its conjugate

$$
\overline{\mathcal{Z}}^{\Lambda}:=\Xi^{\Lambda \Pi^{*}}\left(\mathcal{Z}_{\Pi}\right)^{\dagger}=\left(\begin{array}{c}
\bar{\Upsilon}^{\underline{\alpha}} \\
i \bar{\eta}^{i}
\end{array}\right)=\left(\begin{array}{c}
\bar{\mu}^{\alpha} \\
-\bar{\lambda}_{\dot{\alpha}} \\
i \bar{\eta}^{i}
\end{array}\right)
$$

subject to the so-called (super-)helicity constraint

$$
\mathcal{Z}_{\Lambda} \overline{\mathcal{Z}}^{\Lambda}:=\Upsilon_{\underline{\alpha}} \bar{\Upsilon}^{\underline{\alpha}}+i \eta_{i} \bar{\eta}^{i}=\lambda_{\alpha} \bar{\mu}^{\alpha}-\mu^{\dot{\alpha}} \bar{\lambda}_{\dot{\alpha}}+i \eta_{i} \bar{\eta}^{i}=0
$$

The relation between supertwistors and the variables of the spinor moving frame action (5.18) is given by the Ferber-Penrose incidence relations [68]

$$
\mu^{\dot{\alpha}}=\lambda_{\alpha}\left(X^{\alpha \dot{\alpha}}+2 i \theta_{i}^{\alpha} \sigma^{a} \bar{\theta}^{\dot{\alpha} i}\right)=\left(X^{a}+i \theta_{i} \sigma^{a} \bar{\theta}^{i}\right) \tilde{\sigma}_{a}^{\dot{\alpha} \alpha} \lambda_{\alpha}, \quad \eta_{i}=2 \theta_{i}^{\alpha} \lambda_{\alpha},
$$

supplemented by

$$
\lambda_{\alpha}=\sqrt{\rho^{\#}} v_{\alpha}^{-}, \quad \bar{\lambda}_{\dot{\alpha}}=\sqrt{\rho^{\#}} \bar{v}_{\dot{\alpha}}^{-} .
$$

Notice that (5.22) provides the general solution of (5.21).

Now it is easy to check (see e.g. [42]) that the action (5.18) is equivalent to

$$
S_{\mathrm{smf}}^{4 D}=-\int_{\mathcal{W}^{2}} d^{2} \xi\left(\bar{\partial} \lambda_{\alpha} \bar{\mu}^{\alpha}-\bar{\partial} \mu^{\dot{\alpha}} \bar{\lambda}_{\dot{\alpha}}+i \bar{\partial} \eta_{i} \bar{\eta}^{i}\right)=-\int_{\mathcal{W}^{2}} d^{2} \xi \bar{\partial} \mathcal{Z}_{\Lambda} \overline{\mathcal{Z}}^{\Lambda}
$$

\footnotetext{
${ }^{8}$ The set of our light-like moving frame 4 -vectors can be recognized as Newmann-Penrose or isotropic tetrade of [70-72], $l_{a}=u_{a}^{=}, n_{a}=u_{a}^{\#}, m_{a}=(\bar{m})^{a}=u_{a}^{+-}=\left(u_{a}^{+-}\right)^{*}$; the spinor moving frame variables (called Lorentz harmonics in [47]) can be identified with diads, $\left(v_{\alpha}^{+}, v_{\alpha}^{-}\right)=\left(o_{\alpha}, \imath_{\alpha}\right)$ (see [10-13]). In (5.16), to write compact expressions we denoted $u_{a}^{\#}=: u_{a}^{++}$and $u_{a}^{=}=: u_{a}^{--}$. There and below $v^{ \pm} \sigma_{a} \bar{v}^{ \pm}=v^{\alpha \pm} \sigma_{\alpha \dot{\alpha}}^{a} \bar{v}^{\dot{\alpha} \pm}$, $v^{-} \sigma_{a} \bar{v}^{+}=v^{\alpha-} \sigma_{\alpha \dot{\alpha}}^{a} \bar{v}^{\dot{\alpha}+}$.
} 
with the supertwistor variables obeying (5.21). On the other hand, eq. (5.24) coincides with Siegel's proposal for the twistor (super)string action [43]. Thus [42] twistor string can be identified with $4 \mathrm{D}$ null-superstring and also, in the present perspective, with the $\mathrm{D}=4$ version of the ambitwistor string model of [1].

The special interest to the $\mathcal{N}=4$ version of the twistor string model was related to the fact that the superspace spanned by $\mathcal{N}=4$ supertwistors allows for the existence of the holomorphic integral measure

$$
\Omega_{(3 \mid 4)}=\epsilon_{\underline{\alpha} \underline{\beta \gamma} \underline{\delta}} \Upsilon \underline{\alpha} d \Upsilon^{\underline{\beta}} \wedge d \Upsilon \underline{\gamma} \wedge d \Upsilon^{\underline{\delta}} \epsilon^{i j k l} \frac{\partial}{\partial \eta^{i}} \frac{\partial}{\partial \eta^{j}} \frac{\partial}{\partial \eta^{k}} \frac{\partial}{\partial \eta^{l}}
$$

invariant under the natural U(1) gauge transformations acting on supertwistors. However, in the perspective of ambitwistor string proposal of [1] neither this, nor the spacetime superconformal symmetry of the $\mathrm{D}=4$ supertwistor action does play central role, as it has been oriented on description of amplitudes of 10D SYM, which is not conformally invariant.

However, the classical equivalence of the $\mathrm{D}=4 \mathcal{N}=4$ ambitwistor string to the twistor string which is known to be consistent [44, 45] (at least in tree approximation) suggests that this is the case also for the former, and thus gives us one more argument in favor of the conjecture that ambitwistor string exists and is consistent in a spacetime of any dimensions.

Below we describe the $\mathrm{D}=10$ twistor string model which is classically equivalent to the 10D supersymmetric ambitwistor string of [1], and also $\mathrm{D}=11$ twistor string model which describes the 11D generalization of the ambitwistor string. The arguments of the previous sections allow us to expect that this latter should be consistent and, upon application of the quantization method similar to the one(s) used in $[1,5,6]$, should generate the tree and oneloop amplitudes of $11 \mathrm{D}$ supergravity. Actually, we prefer to begin with the $\mathrm{D}=11$ model.

\subsection{Twistor string in $\mathrm{D}=11$ and $\mathrm{D}=10$ from ambitwistor/null superstring action}

To make manifest the twistor-like structure of the moving fame action (3.13) of $\mathrm{D}=11$ or $\mathrm{D}=10$ null superstring, which, as we have shown, is classically equivalent to ambitwistor superstring, we have to substitute the expression for $u_{a}^{=}$in terms of spinor bilinear which follow from the constraints (5.5) or (5.12).

\subsubsection{Spinor moving frame action for 11D ambitwistor superstring and its reformulation in the generalized 11D superspace}

In $\mathrm{D}=11$ it is convenient, following [50], to introduce

$$
\lambda_{\alpha q}=\sqrt{\rho^{\#}} v_{\alpha}^{-q},
$$

which, as a consequence of (5.5) obey ${ }^{9}$

$$
\lambda_{q} \tilde{\Gamma}_{a} \lambda_{p}=P_{a} \delta_{q p}, \quad 2 \lambda_{\alpha q} \lambda_{\beta q}=\Gamma_{\alpha \beta}^{a} P_{a}, \quad P_{a} P^{a}=0 .
$$

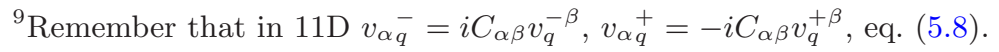


Here the light-like vector $P_{a}$ originates in the product of the Lagrange multiplier and the moving frame vector, $P_{a}=\rho^{\#} u_{a}^{\overline{=}}$, cf. eq. (3.4).

The relation with spinor moving frame, (5.26), guarantees the consistency of the constraints (5.27) (which probably is not so apparent).

Notice also that all the constraints (5.27), as well as all the expressions below, are invariant under $\mathrm{SO}(16)$ transformations acting on the indices $q, p=1, \ldots, 16$, so that we can solve the constraints by

$$
\lambda_{\alpha q}=\sqrt{\rho^{\#}} v_{\alpha}^{-p} S_{p q}, \quad S_{p p^{\prime}} S_{q p^{\prime}}=\delta_{p q} .
$$

Now substituting $\frac{1}{32} \lambda_{\alpha q} \lambda_{\beta q} \tilde{\Gamma}^{a \alpha \beta}$ for $\rho^{\#} u_{a}^{=}$in (3.13) we obtain

$$
\begin{aligned}
S_{\mathrm{smf}}^{11 D} & =\int_{\mathcal{W}^{2}} d^{2} \xi \lambda_{\alpha q} \lambda_{\beta q} \frac{1}{32} \tilde{\Gamma}_{a}^{\alpha \beta}\left(\bar{\partial} X^{a}-i \bar{\partial} \Theta \Gamma^{a} \Theta\right)= \\
& =\int_{\mathcal{W}^{2}} d^{2} \xi \lambda_{\alpha q} \lambda_{\beta q}\left(\bar{\partial} X^{\alpha \beta}-i \bar{\partial} \Theta^{(\alpha} \Theta^{\beta)}\right) .
\end{aligned}
$$

Here to pass to the second form of the action we have defined

$$
X^{\alpha \beta}=\frac{1}{32} X^{a} \tilde{\Gamma}_{a}^{\alpha \beta}
$$

and have used the Fiertz identity

$$
\frac{1}{32} \tilde{\Gamma}_{a}^{\alpha \beta} \bar{\partial} \Theta \Gamma^{a} \Theta=\bar{\partial} \Theta^{(\alpha} \Theta^{\beta)}+\frac{1}{64} \tilde{\Gamma}_{a b}^{\alpha \beta} \bar{\partial} \Theta \Gamma^{a b} \Theta-\frac{1}{32 \cdot 5 !} \tilde{\Gamma}_{a b c d e}^{\alpha \beta} \bar{\partial} \Theta \Gamma^{a b c d e} \Theta
$$

to simplify the second term in the bracket. Notice that, as a consequence of (5.27), after contraction with $\lambda_{\alpha q} \lambda_{\beta q}$ the second and the third terms in (5.31) do not contribute so that the net result is provided by the first term, $\bar{\partial} \Theta^{(\alpha} \Theta^{\beta)}$.

Furthermore, due to the same reasons one can consider $X^{\alpha \beta}$ in (5.29) as generic symmetric matrix. Indeed, the general decomposition of such a $32 \times 32$ symmetric matrix

$$
X^{\alpha \beta}=\frac{1}{32} X^{a} \tilde{\Gamma}_{a}^{\alpha \beta}-\frac{1}{64} \tilde{\Gamma}_{a b}^{\alpha \beta} Z^{a b}+\frac{1}{32 \cdot 5 !} \tilde{\Gamma}_{a b c d e}^{\alpha \beta} Z^{a b c d e}
$$

contains the contributions of "tensorial central charge coordinates" $Z^{a b}=-Z^{b a}=Z^{[a b]}$ and $Z^{a b c d e}=Z^{[a b c d e]}$. However, these do not produce any contribution into the action (5.29) because, as a consequence of (5.27),

$$
\lambda_{q} \tilde{\Gamma}_{a b} \lambda_{q}=0, \quad \lambda_{q} \tilde{\Gamma}_{a b c d e} \lambda_{q}=0,
$$

(cf. with the discussion of 11D superparticle action in [50]).

Hence, interestingly enough, the twistor-like spinor moving frame formulation of ambitwistor/null superstring allows to treat it as a dynamical system in the enlarged superspace of 528 bosonic and 32 fermionic dimensions, $\Sigma^{(528 \mid 32)}$ with coordinates $X^{\alpha \beta}$ and $\Theta^{\alpha}$. This enlarged superspace was discussed for the first time in [81]. Various dynamical system in this superspace were studied in $[49,82-84,86]$. Notice that it is also related with hidden gauge symmetry structure of $11 \mathrm{D}$ supergravity [87-89] and with notion of BPS preon $[85,90]$; this allows us to hope on its possible significance in the ambitwistor/twistor string context. 


\subsubsection{D twistor string action from ambitwistor superstring}

Moving the derivative $\bar{\partial}$ we can write the 11D null/ambitwistor superstring action (5.29) in the form characteristic for Siegel's twistor string,

$$
\begin{aligned}
S_{\mathrm{twS}}^{11 D} & =-\int_{\mathcal{W}^{2}}\left(\bar{\partial} \lambda_{\alpha q} \mu_{q}^{\alpha}-\lambda_{\alpha q} \bar{\partial} \mu_{q}^{\alpha}+i \bar{\partial} \eta_{q} \eta_{q}\right)= \\
& =-\int_{\mathcal{W}^{2}} \bar{\partial} \mathcal{Z}_{\Lambda q} \Xi^{\Lambda \Sigma} \mathcal{Z}_{\Sigma q}, \quad \Xi^{\Lambda \Sigma}=\left(\begin{array}{cccc}
0 & \delta^{\alpha}{ }_{\beta} & 0 \\
-\delta_{\alpha}{ }^{\beta} & 0 & 0 \\
0 & 0 & i
\end{array}\right) .
\end{aligned}
$$

The dynamical variables of this action, real bosonic spinors $\lambda_{\alpha q}$ and $\mu_{q}^{\alpha}$, and real fermionic scalars $\eta_{q}$ can be collected in 16 strongly constrained $\operatorname{OSp}(1 \mid 64)$ supertwistors $^{10}$

$$
\mathcal{Z}_{\Lambda q}:=\left(\Upsilon_{\underline{\alpha} q} ; \eta_{q}\right)=\left(\lambda_{\alpha q}, \mu_{q}^{\alpha} ; \eta_{q}\right), \quad \alpha=1, \ldots, 32, \quad q=1, \ldots, 16 .
$$

They are expressed through the coordinate functions and spinor moving frame variables by the following generalization of the Penrose incidence relations

$$
\mu_{q}^{\alpha}=X^{\alpha \beta} \lambda_{\beta q}-\frac{i}{2} \Theta^{\alpha} \Theta^{\beta} \lambda_{\beta q}, \quad \eta_{q}=\Theta^{\beta} \lambda_{\beta q} .
$$

These relations with generic $X^{\alpha \beta}=X^{\beta \alpha}$ (5.32) and $\lambda_{\alpha q}$ obeying (5.27),

$$
\lambda_{q} \tilde{\Gamma}_{a} \lambda_{p}=P_{a} \delta_{q p}, \quad 2 \lambda_{\alpha q} \lambda_{\beta q}=\Gamma_{\alpha \beta}^{a} P_{a}, \quad P_{a}:=\frac{1}{16} \lambda_{q} \tilde{\Gamma}_{a} \lambda_{q},
$$

provide us with the general solution of the constraints (see [50])

$$
\mathbb{J}_{p q}=\mathcal{Z}_{\Lambda[p} \Xi^{\Lambda \Sigma} \mathcal{Z}_{q] \Sigma}=2 \lambda_{\alpha[p} \mu_{q]}^{\alpha}+i \eta_{p} \eta_{q}=0 .
$$

Expression (5.37) with particular $X^{\alpha \beta}$ expressed through the standard 11D spacetime coordinate, (5.30), appears if we impose, in addition to (5.39) (5.38), also the constraint

$$
\mathbb{K}_{p q}=\lambda_{\alpha(p} \mu_{q)}^{\alpha}-\frac{1}{16} \delta_{p q} \lambda_{\alpha p^{\prime}} \mu_{p^{\prime}}^{\alpha}=0 .
$$

Clearly, this breaks the $\operatorname{OSp}(1 \mid 64)$ invariance which might be attributed to the action (5.34), (5.35) and to the constraint (5.39). To write (5.40) in terms of the whole supertwistor (5.36) we have to use the degenerate symmetric matrix

$$
G^{\Lambda \Sigma}=\left(\begin{array}{ccc}
0 & \delta^{\alpha}{ }_{\beta} & 0 \\
\delta_{\alpha}{ }^{\beta} & 0 & 0 \\
0 & 0 & 0
\end{array}\right) ; \quad \mathbb{K}_{p q}=\mathcal{Z}_{\Lambda[p} G^{\Lambda \Sigma} \mathcal{Z}_{q] \Sigma}=0
$$

This matrix is preserved by bosonic GL(32) subgroup of OSp(1|64) supergroup only. But even this invariance is actually broken already by the constraints (5.38) imposed on the first, $\lambda$-component of the supertwistor.

\footnotetext{
${ }^{10}$ Generically such supertwistors with 64 bosonic and 1 fermionic components provide the fundamental representation of the $\operatorname{OSp}(1 \mid 64)$ supergroup. The $\operatorname{OSp}(1 \mid 64)$ transformations leave invariant the matrix $\Xi^{\Lambda \Sigma}$ defined in (5.35). See $[83,86]$ for more details.
} 
Notice that

$$
\begin{aligned}
\mathcal{I}_{+}^{\Lambda \Sigma}:=\frac{1}{2}\left(G^{\Lambda \Sigma}+\Sigma^{\Lambda \Sigma}\right) & =\left(\begin{array}{ccc}
0 & 0 & 0 \\
\delta_{\alpha}{ }^{\beta} & 0 & 0 \\
0 & 0 & i
\end{array}\right) \\
\text { or } \quad \mathcal{I}_{-}^{\Lambda \Sigma}: & :=\frac{1}{2}\left(G^{\Lambda \Sigma}-\Sigma^{\Lambda \Sigma}\right)=\left(\begin{array}{ccc}
0 & \delta^{\alpha} & 0 \\
0 & 0 & 0 \\
0 & 0 & -i
\end{array}\right)
\end{aligned}
$$

can be considered as a counterpart of $\mathrm{D}=4$ 'infinite twistor' $\mathcal{I}_{+}^{\underline{\alpha} \underline{\beta}}=\left(\begin{array}{cc}0 & 0 \\ 0 & \epsilon^{\dot{\alpha} \dot{\beta}}\end{array}\right)$ or $\mathcal{I}_{-}^{\underline{\alpha} \underline{\beta}}=$ $\left(\begin{array}{cc}\epsilon^{\alpha \beta} & 0 \\ 0 & 0\end{array}\right)[71,72]$ (see also e.g. [80]).

Although our supertwistors are strongly constrained, their relation with spinor moving frame variables, eq. (5.23), allows to define variational problem in a simple way. The most essential is the variation of $\lambda_{\alpha q}$ which can be written in the form of (see $[51,52,78]$ for details)

$$
\delta \lambda_{\alpha q}=i_{\delta} f^{(0)} \lambda_{\alpha q}+\lambda_{\alpha p} i_{\delta} f^{[p q]}+\frac{1}{2} i_{\delta} f^{-i} v_{\alpha p}{ }^{+} \gamma_{p q^{\prime}}^{i} S_{q^{\prime} q}
$$

where $i_{\delta} f^{(0)}, i_{\delta} f^{[p q]}=-i_{\delta} f^{[q p]}$ and $i_{\delta} f^{-i}\left(=\sqrt{\rho^{\#}} i_{\delta} \Omega^{=i}\right.$ in the notation of $\left.[51,52,78]\right)$ denote independent variations and the last term refers explicitly to the solution (5.28) of the constraints (5.27): in it $S_{p q}=S_{q p}^{-1}$ is the $\mathrm{SO}(16)$-valued matrix field, $v_{\alpha p}{ }^{+}$are the spinor moving frame variables complementary to $v_{\alpha p}{ }^{-}$(see (5.2), (5.11), (5.12), (5.13)) and $\gamma_{p q}^{i}=\gamma_{q p}^{i}$ are $\mathrm{SO}(9)$ invariant gamma matrices.

The twistor-like formulation of 11D massless superparticle model, which can be obtained from our twistor superstring by replacing worldsheet $\mathcal{W}^{2}$ by a worldline, making all the field dependent on one proper time variable $\tau$ instead of two worldsheet coordinates, and replacing $\bar{\partial}$ by $\partial_{\tau}$, was discussed in [50], where it was shown that its quantization gives the linearized 11D supergravity multiplet. This allows us to hope that the quantization of the spinor moving frame formulation of the ambitwistor string model along the lines of $[1,6]$ and/or [39-41] can produce the amplitudes of the 11D supergravity.

\subsubsection{D twistor string action from ambitwistor superstring}

The 10D version of the ambitwistor superstring action can also be rewritten in twistor-like (spinor moving frame) form and as a twistor string action for a set of strongly constrained twistors. ${ }^{11}$ The equations are very similar to the ones for $11 \mathrm{D}$ ambitwistor string, up to that the spinor indices $\alpha, \beta$ are now taking 16 values (not 32 as in $\mathrm{D}=11$ ), and the basic set of bosonic spinor carries the dotted, c-spinor index of $\mathrm{SO}(8), \dot{q}=1, \ldots, 8$. This allows us to be short in this section.

\footnotetext{
${ }^{11}$ See $[73-75]$ for the discussion on twistor transform of tensionful superstrings in $\mathrm{D}=3,4,6,10$ and $[76,77]$ for related studies.
} 
Using the $\mathrm{D}=10$ spinor moving frame variables we introduce the set of 8 composed Majorana-Weyl spinors,

$$
\lambda_{\alpha \dot{q}}=\sqrt{\rho^{\#}} v_{\alpha \dot{p}}-S_{\dot{p} \dot{q}}, \quad S S^{T}=\mathbb{I}_{8 \times 8}, \quad \alpha, \beta=1, \ldots, 16
$$

which solves the constraints (cf. (5.27))

$$
\lambda_{\dot{q}} \tilde{\sigma}_{a} \lambda_{\dot{p}}=p_{a} \delta_{\dot{q} \dot{p}}, \quad 2 \lambda_{\alpha \dot{q}} \lambda_{\beta \dot{q}}=\Gamma_{\alpha \beta}^{a} p_{a},
$$

involving a ten vector field $p_{a}$. This is light-like, $p_{a} p^{a}=0$, as can be deduced from the identity $\tilde{\sigma}^{a \alpha(\beta} \tilde{\sigma}_{a}^{\gamma \delta)}=0$ (in $\mathrm{D}=11$, there is no such type identity, and the proof of lightlikeness of $P_{a}$ in (5.46) requires to use the charge conjugation matrix, see e.g. [78]).

The spinor moving frame action for $10 \mathrm{D}$ twistor string reads

$$
\begin{aligned}
S_{\mathrm{smf}}^{10 D} & =\int_{\mathcal{W}^{2}} d^{2} \xi \lambda_{\alpha \dot{q}} \lambda_{\beta \dot{q}} \frac{1}{16} \tilde{\sigma}_{a}^{\alpha \beta}\left(\bar{\partial} X^{a}-i \bar{\partial} \Theta \sigma^{a} \Theta\right)= \\
& =\int_{\mathcal{W}^{2}} d^{2} \xi \lambda_{\alpha \dot{q}} \lambda_{\beta \dot{q}}\left(\bar{\partial} X^{\alpha \beta}-i \bar{\partial} \Theta^{(\alpha} \Theta^{\beta)}\right),
\end{aligned}
$$

where in the second line $16 \times 16$ matrix field $X^{\alpha \beta}=X^{\beta \alpha}$ can be considered as constructed from the 10-vector coordinate function,

$$
X^{\alpha \beta}=\frac{1}{16} X^{a} \tilde{\sigma}_{a}^{\alpha \beta},
$$

or to be a generic symmetric spin-tensor field

$$
X^{\alpha \beta}=\frac{1}{16} X^{a} \tilde{\sigma}_{a}^{\alpha \beta}+\frac{1}{32 \cdot 5 !} \tilde{\sigma}_{a b c d e}^{\alpha \beta} Z^{a b c d e}
$$

which contains the contributions of 5-rank self-dual tensorial $Z^{\text {abcde }}=$ $\frac{1}{5 !} \epsilon^{a b c d e a^{\prime} b^{\prime} c^{\prime} d^{\prime} e^{\prime} f^{\prime}} Z_{a^{\prime} b^{\prime} c^{\prime} d^{\prime} e^{\prime} f^{\prime}}$. This latter does not contribute to the action because the bosonic spinor $\lambda_{\dot{q}}$ in (5.45) obeys

$$
\lambda_{\dot{q}} \tilde{\sigma}_{a b c d e} \lambda_{\dot{q}}=0 .
$$

As in eleven-dimensional case, the spinor moving frame action of 10D ambitwistor/null string (5.47) can be equivalently written as twistor string action

$$
S_{10 D}^{\mathrm{twS}}=-\int_{\mathcal{W}^{2}}\left(\bar{\partial} \lambda_{\alpha \dot{q}} \mu^{\alpha \dot{q}}-\lambda_{\alpha \dot{q}} \bar{\partial} \mu^{\alpha \dot{q}}+i \bar{\partial} \eta_{\dot{q}} \eta_{\dot{q}}\right)=-\int_{\mathcal{W}^{2}} \bar{\partial} \mathcal{Z}_{\Lambda \dot{q}} \Xi^{\Lambda \Sigma} \mathcal{Z}_{\Sigma \dot{q}}
$$

where $\Xi^{\Lambda \Sigma}$ has the same form as in (5.35), but with $16 \times 16$ blocks $\delta_{\alpha}^{\beta}$.

The action (5.51) is written in terms of a set of 8 strongly constrained $\operatorname{OSp}(1 \mid 32)$ supertwistors

$$
\mathcal{Z}_{\Lambda \dot{q}}:=\left(\Upsilon_{\underline{\alpha} \dot{q}} ; \eta_{\dot{q}}\right)=\left(\lambda_{\alpha \dot{q}}, \mu_{\dot{q}}^{\alpha} ; \eta_{\dot{q}}\right), \quad \alpha=1, \ldots, 16, \quad \dot{q}=1, \ldots, 8
$$

which can be expressed through the 10D coordinate functions and spinor moving frame variables by the following generalization of the Penrose incidence relation

$$
\mu^{\alpha \dot{q}}=X^{\alpha \beta} \lambda_{\beta \dot{q}}-\frac{i}{2} \Theta^{\alpha} \Theta^{\beta} \lambda_{\beta \dot{q}}, \quad \eta_{q}=\Theta^{\beta} \lambda_{\beta \dot{q}} .
$$


In addition to (5.46), the supertwistor components obey the constraint

$$
\mathbb{J}_{\dot{p} \dot{q}}=\mathcal{Z}_{\Lambda[\dot{p}} \Xi^{\Lambda \Sigma} \mathcal{Z}_{\dot{q}] \Sigma}=2 \lambda_{\alpha[\dot{p}} \mu_{\dot{q}]}^{\alpha}+i \eta_{\dot{p}} \eta_{\dot{q}}=0,
$$

and, in the case of $X^{\alpha \beta}$ expressed through $X^{a}$ by (5.49), also

$$
\mathbb{K}_{\dot{p} \dot{q}}=\lambda_{\alpha(\dot{p}} \mu_{\dot{q})}^{\alpha}-\frac{1}{16} \delta_{\dot{p} \dot{q}} \lambda_{\alpha \dot{p}^{\prime}} \mu_{\dot{p}^{\prime}}^{\alpha}=0 .
$$

These relations with generic $X^{\alpha \beta}=X^{\beta \alpha}$ (5.32) and $\lambda_{\alpha \dot{q}}$ obeying the constraints (5.46) provide us with the general solution of the constraints (see [50])

$$
\mathbb{J}_{\dot{p} \dot{q}}=\mathcal{Z}_{\Lambda[\dot{p}} \Xi^{\Lambda \Sigma} \mathcal{Z}_{\dot{q}] \Sigma}=2 \lambda_{\alpha[\dot{p}} \mu_{\dot{q}]}^{\alpha}+i \eta_{\dot{p}} \eta_{\dot{q}}=0
$$

Eq. (5.37) with particular $X^{\alpha \beta}$ expressed through the standard 11D spacetime coordinate, (5.30), appears if we impose, in addition to (5.39) (5.38), also the constraints

$$
\mathbb{K}_{\dot{p} \dot{q}}=\lambda_{\alpha(\dot{p}} \mu_{\dot{q})}^{\alpha}-\frac{1}{16} \lambda_{\alpha \dot{r}} \mu_{\dot{r}}^{\alpha} \delta_{\dot{p} \dot{q}}=0 .
$$

The twistor string is classically equivalent to the 10D ambitwistor superstring of [1], (2.2) [as its action (5.51) was obtained from (2.2) by solving the algebraic constraints and changing the variables]. We can show that the quantization of its superparticle limit gives the 10D SYM theory. As a result, we can expect that its quantization in the twistor string line of [39] or in the line of [1] can produce the amplitudes of the 10D SYM.

Such a quantization is a natural continuation of our study. An apparent technical problem on this way is the constrained nature of our supertwistors. However, it can be resolved using their relation with the spinor moving frame variables: this allows to define the admissible variation of the $\lambda$-spinor which preserve the constraints (5.46) which can be written as (cf. with (5.44) of the $\mathrm{D}=11$ case)

$$
\delta \lambda_{\alpha \dot{q}}=i_{\delta} f^{(0)} \lambda_{\alpha \dot{q}}+\lambda_{\alpha \dot{p}} i_{\delta} f^{[\dot{p} \dot{q}]}+\frac{1}{2} i_{\delta} f^{-i} v_{\alpha p}{ }^{+} \gamma_{p \dot{s}}^{i} S_{\dot{s} \dot{q}}
$$

Here $i_{\delta} f^{(0)}, i_{\delta} f^{[p q]}=-i_{\delta} f^{[q p]}$ and $i_{\delta} f^{-i}$ denote independent variations and the last term refers explicitly to the solution (5.45) of the constraints (5.27): $S_{\dot{p} \dot{q}}=S_{\dot{q} \dot{p}}^{-1}$ is the matrix field taking values in c-spinor representation of $\mathrm{SO}(8), v_{\alpha p}{ }^{+}$are the spinor moving frame variables complementary to $v_{\alpha \dot{p}}{ }^{-}$(see (5.3), (5.11), (5.12), (5.13)) and $\gamma_{p \dot{q}}^{i}=\tilde{\gamma}_{\dot{q} p}^{i}$ are $\mathrm{SO}(8)$ invariant gamma matrices.

\section{Conclusion}

In this paper we first have shown that $\mathrm{D}=4,10$ and $\mathrm{D}=11$ versions of the recently proposed (for $\mathrm{D}=10$ ) ambitwistor superstring $[1]^{12}$ is classically equivalent to the null-superstring in its moving frame and spinor moving frame formulations [10-13].

\footnotetext{
${ }^{12}$ The $\mathrm{D}=26$ purely bosonic ambitwistor string and the $\mathrm{D}=10$ NSR version of ambitwistor string were also considered in [1].
} 
The null-string and null-superstring is usually associated to tensionless limit of NambuGoto (NG) string and Green-Schwarz (GS) superstring. In contrast, the ambitwistor (super)string was associated in [1] with infinite tension limit of the NG (GS super)string. However, on the other hand, the authors of [1] noticed the similarity of the properties of the ambitwistor string amplitudes with the very high energy limit of string amplitudes [7, 8], which is naturally associated to the tensionless limit of superstring.

To clarify this issue in this paper we have shown how the null-superstring action can dominate both the tensionless and infinite tension limit of the superstring action. Furthermore, the tensionless limit of superstring theory does not have critical dimensions, this is to say, it can be formulated in any $\mathrm{D}[18,22,36-38]$. In this paper we have presented arguments which suggest that this is also true in the limit of infinite tension. (Actually this conjecture looks quite natural as in the infinite tension limit string is expected to behave like a particle).

This conjecture is further supported by the observation that the spinor moving frame formulation of $\mathrm{D}=4, \mathcal{N}$-extended supersymmetric null-superstring, which is classically equivalent to $\mathrm{D}=4$ version of the ambitwistor string, is also classically equivalent to Siegel's formulation of closed twistor superstring [43]. The twistor string was originally formulated for $\mathrm{D}=4 \mathcal{N}=4$ case, in which the twistor superspace is Calabi-Yau supermanifold [44, 45], and is known to be a consistent theory (at least at the tree level, see [79, 80] and refs. therin).

The similar twistor transform allows us to present the spinor moving frame formulation of D-dimensional null superstring, classically equivalent to D-dimensional version of the ambitwistor string, as a D-dimensional twistor string. Besides $\mathrm{D}=4$, we have described the $\mathrm{D}=10$ twistor string, classically equivalent to the original 10D ambitwistor superstring action of [1], and the $\mathrm{D}=11$ twistor superstring. Both $\mathrm{D}=10$ and $\mathrm{D}=11$ models are formulated in terms of highly constrained $\operatorname{OSp}(1 \mid 2 n)$ supertwistors $(n=16$ for $\mathrm{D}=10$ and $n=32$ for $\mathrm{D}=11$ ). However, the relation of the component of supertwistors with spinor moving frame variables allows us to present simple expressions for their admissible variations which preserve the constraints. The quantization of the 11D null-superstring/twistor string model in the line of [1] or [39] is expected to produce amplitudes of 11D supergravity. To develop such a quantization and to obtain the $11 \mathrm{D}$ amplitudes is an interesting task for future study.

Interestingly enough, the generalized Ferber-Penrose (FP) incidence relations expressing the supertwistors through the coordinate functions and spinor moving frame variables describing the ambitwistor string (null-superstring) are gauge equivalent to a more general FP relations involving additional coordinates of enlarged or tensorial superspace, $\Sigma^{(528 \mid 32)}$ parametrized by $X^{a}, Z^{a b}=Z^{[a b]}$ and $Z^{a b c d e}=Z^{[a b c d e]}$ in $11 \mathrm{D}$ case. The gauge symmetry of the twistor like formulation of the ambitwistor string and of the twistor superstring action, which allow to gauge away the additional $517(=55+462)$ coordinates $Z^{a b}$ and $Z^{a b c d e}=Z^{[a b c d e]}$ seems to be related with the hidden gauge symmetry structure of 11D supergravity [87-89]. This makes tempting to speculate on possible relevance of the enlarged 'tensorial' superspaces in the ambitwistor string context.

In the recent paper [91] the authors, approaching the scattering equation of [2] in the context of (standard) string theory, formulated a new 'dual resonance' model which 
coincides with string theory in both the $\alpha^{\prime} \mapsto 0$ and $\alpha^{\prime} \mapsto \infty$ limits. They observed that the solutions of this dual model can be found algebraically on the surface of scattering equations producing the $\alpha^{\prime}$ corrections to the amplitudes, checked that these coincide with known results in several previously studied cases. The authors of [91] have stress that, despite they worked according the rules of string theory, their dual model cannot by the usual string theory.

In the light of our present results, an interesting question for future study is whether the dual model of [91] can be related to (10D verison of) twistor string which, as we have shown, is classically equivalent to null superstring and ambitwistor string.

Note added. After this paper appeared on the net, the ambitwistor string technique have been applied to the $\mathrm{D}=4$ case in [92] and used there to obtain the expressions for $\mathcal{N}$-extended SYM and Einstein supergravity amplitudes. The authors of [92] reformulated the ambitwistor string model in terms of supertwistors, similarly to what had been done in sections 3.1, 5.1, 5.2 above, and noticed that their theory coincides with ("is superficially identical to") twistor string, thus agreeing with our conclusion in section 5.2. On the other side they have stressed that the resulting formulae for amplitudes are different from that following from twistor string approach as formulated in [44, 45, 79]. The study of [92] confirms the conclusion of this paper on the existence of generalizations of ambitwistor string technique $[1,5]$ for arbitrary spacetime dimensions.

\section{Acknowledgments}

The author thanks Dima Sorokin for numerous useful discussions and communications, and also for hospitality in Padova University and Padova section of INFN, where a part of this work was done. It is also a pleasure to thank Nicolya Boulanger for discussions on $[57,58]$ and $[41]$, which renewed his interest to the twistor string approach. A partial support from the Spanish MINECO research grants FPA2012-35043-C02-01, from the Basque Government Research Group Grant ITT559-10 and from UPV/EHU under the program UFI $11 / 55$ is greatly acknowledged.

Open Access. This article is distributed under the terms of the Creative Commons Attribution License (CC-BY 4.0), which permits any use, distribution and reproduction in any medium, provided the original author(s) and source are credited.

\section{References}

[1] L. Mason and D. Skinner, Ambitwistor strings and the scattering equations, JHEP 07 (2014) 048 [arXiv: 1311.2564] [INSPIRE].

[2] F. Cachazo, S. He and E.Y. Yuan, Scattering of Massless Particles in Arbitrary Dimension, arXiv:1307.2199 [INSPIRE].

[3] L. Dolan and P. Goddard, Proof of the Formula of Cachazo, He and Yuan for Yang-Mills Tree Amplitudes in Arbitrary Dimension, JHEP 05 (2014) 010 [arXiv:1311.5200] [INSPIRE]. 
[4] R. Britto, F. Cachazo, B. Feng and E. Witten, Direct proof of tree-level recursion relation in Yang-Mills theory, Phys. Rev. Lett. 94 (2005) 181602 [hep-th/0501052] [INSPIRE].

[5] T. Adamo, E. Casali and D. Skinner, Ambitwistor strings and the scattering equations at one loop, JHEP 04 (2014) 104 [arXiv: 1312.3828] [INSPIRE].

[6] N. Berkovits, Infinite Tension Limit of the Pure Spinor Superstring, JHEP 03 (2014) 017 [arXiv: 1311.4156] [INSPIRE].

[7] D.J. Gross and P.F. Mende, The High-Energy Behavior of String Scattering Amplitudes, Phys. Lett. B 197 (1987) 129 [INSPIRE].

[8] D.J. Gross and P.F. Mende, String Theory Beyond the Planck Scale, Nucl. Phys. B 303 (1988) 407 [INSPIRE].

[9] I.A. Bandos and A.A. Zheltukhin, Hamiltonian mechanics and absence of critical dimensions for null membranes, Sov. J. Nucl. Phys. 50 (1989) 556 [INSPIRE].

[10] I.A. Bandos and A.A. Zheltukhin, Twistors, harmonics and zero super-p-branes, JETP Lett. 51 (1990) 618 [INSPIRE].

[11] I.A. Bandos and A.A. Zheltukhin, Quantum theory of closed null supermembranes in four-dimensional space, JETP Lett. 53 (1991) 5 [INSPIRE].

[12] I.A. Bandos and A.A. Zheltukhin, Null super p-brane: Hamiltonian dynamics and quantization, Phys. Lett. B 261 (1991) 245 [INSPIRE].

[13] I.A. Bandos and A.A. Zheltukhin, Null super p-branes quantum theory in four-dimensional space-time, Fortsch. Phys. 41 (1993) 619 [InSPIRE].

[14] A. Schild, Classical Null Strings, Phys. Rev. D 16 (1977) 1722 [inSPIRE].

[15] A. Karlhede and U. Lindström, The Classical Bosonic String in the Zero Tension Limit, Class. Quant. Grav. 3 (1986) L73 [InSPIRE].

[16] A.A. Zheltukhin, A Hamiltonian of null strings: an invariant action of null (super)membranes, Sov. J. Nucl. Phys. 48 (1988) 375 [INSPIRE].

[17] J. Gamboa, C. Ramirez and M. Ruiz-Altaba, Quantum null (super)strings, Phys. Lett. B 225 (1989) 335 [INSPIRE].

[18] U. Lindström, B. Sundborg and G. Theodoridis, The zero tension limit of the superstring, Phys. Lett. B 253 (1991) 319 [INSPIRE].

[19] P. Bozhilov, $N=1, D=10$ tensionless superbranes, Phys. Lett. B 440 (1998) 35 [hep-th/9806134] [INSPIRE].

[20] P. Bozhilov, N=1, D = 10 tensionless superbranes II, Phys. Lett. B 454 (1999) 27 [hep-th/9901153] [INSPIRE].

[21] P. Bozhilov, Null branes in curved backgrounds, Phys. Rev. D 60 (1999) 125011 [hep-th/9904208] [INSPIRE].

[22] U. Lindström and M. Zabzine, Tensionless strings, WZW models at critical level and massless higher spin fields, Phys. Lett. B 584 (2004) 178 [hep-th/0305098] [INSPIRE].

[23] I.A. Bandos and A.A. Zheltukhin, Spinor Cartan moving n hedron, Lorentz harmonic formulations of superstrings and kappa symmetry, JETP Lett. 54 (1991) 421 [INSPIRE].

[24] I.A. Bandos and A.A. Zheltukhin, Green-Schwarz superstrings in spinor moving frame formalism, Phys. Lett. B 288 (1992) 77 [INSPIRE]. 
[25] I.A. Bandos and A.A. Zheltukhin, Twistor-like approach in the Green-Schwarz D $=10$ superstring theory, Phys. Part. Nucl. 25 (1994) 453 [inSPIRE].

[26] E. Sokatchev, Light Cone Harmonic Superspace and Its Applications, Phys. Lett. B 169 (1986) 209 [inSPIRE].

[27] E. Sokatchev, Harmonic superparticle, Class. Quant. Grav. 4 (1987) 237 [INSPIRE].

[28] E.R. Nissimov and S.J. Pacheva, Manifestly SuperPoincaré Covariant Quantization of the Green-Schwarz Superstring, Phys. Lett. B 202 (1988) 325 [INSPIRE].

[29] E. Nissimov, S. Pacheva and S. Solomon, Covariant Canonical Quantization of the Green-Schwarz Superstring, Nucl. Phys. B 297 (1988) 349 [InSPIRE].

[30] E. Nissimov, S. Pacheva and S. Solomon, Off-shell Superspace D = 10 Super Yang-Mills From Covariantly Quantized Green-Schwarz Superstring, Nucl. Phys. B 317 (1989) 344 [INSPIRE].

[31] R. Kallosh and M. Rakhmanov, Covariant Quantization of the Green-Schwarz Superstring, Phys. Lett. B 209 (1988) 233 [inSPIRE].

[32] R. Kallosh and M. Rakhmanov, Consistency of Covariant Quantization of Gs String, Phys. Lett. B 214 (1988) 549 [inSPIRE].

[33] A.S. Galperin, P.S. Howe and K.S. Stelle, The superparticle and the Lorentz group, Nucl. Phys. B 368 (1992) 248 [hep-th/9201020] [INSPIRE].

[34] F. Delduc, A. Galperin and E. Sokatchev, Lorentz harmonic (super)fields and (super)particles, Nucl. Phys. B 368 (1992) 143 [INSPIRE].

[35] A.S. Galperin, P.S. Howe and P.K. Townsend, Twistor transform for superfields, Nucl. Phys. B 402 (1993) 531 [inSPIRE].

[36] D. Francia and A. Sagnotti, On the geometry of higher spin gauge fields, Class. Quant. Grav. 20 (2003) S473 [hep-th/0212185] [INSPIRE].

[37] A. Sagnotti and M. Tsulaia, On higher spins and the tensionless limit of string theory, Nucl. Phys. B 682 (2004) 83 [hep-th/0311257] [INSPIRE].

[38] G. Bonelli, On the tensionless limit of bosonic strings, infinite symmetries and higher spins, Nucl. Phys. B 669 (2003) 159 [hep-th/0305155] [INSPIRE].

[39] Y. Abe, V.P. Nair and M.-I. Park, Multigluon amplitudes, $N=4$ constraints and the WZW model, Phys. Rev. D 71 (2005) 025002 [hep-th/0408191] [INSPIRE].

[40] R. Boels, L.J. Mason and D. Skinner, Supersymmetric Gauge Theories in Twistor Space, JHEP 02 (2007) 014 [hep-th/0604040] [INSPIRE].

[41] T. Adamo, Twistor actions for gauge theory and gravity, arXiv:1308.2820 [INSPIRE].

[42] I.A. Bandos, J.A. de Azcarraga and C. Miquel-Espanya, Superspace formulations of the (super)twistor string, JHEP 07 (2006) 005 [hep-th/0604037] [INSPIRE].

[43] W. Siegel, Untwisting the twistor superstring, hep-th/0404255 [INSPIRE].

[44] E. Witten, Perturbative gauge theory as a string theory in twistor space, Commun. Math. Phys. 252 (2004) 189 [hep-th/0312171] [INSPIRE].

[45] N. Berkovits, An alternative string theory in twistor space for $N=4$ super Yang-Mills, Phys. Rev. Lett. 93 (2004) 011601 [hep-th/0402045] [INSPIRE]. 
[46] O.T. Engelund and R. Roiban, A twistor string for the ABJ(M) theory, JHEP 06 (2014) 088 [arXiv: 1401.6242] [INSPIRE].

[47] I.A. Bandos, Superparticle in Lorentz harmonic superspace (In Russian), Sov. J. Nucl. Phys. 51 (1990) 906 [INSPIRE].

[48] I.A. Bandos and A.Y. Nurmagambetov, Generalized action principle and extrinsic geometry for $N=1$ superparticle, Class. Quant. Grav. 14 (1997) 1597 [hep-th/9610098] [INSPIRE].

[49] I.A. Bandos and J. Lukierski, New superparticle models outside the HLS supersymmetry scheme, Lect. Notes Phys. 539 (2000) 195 [hep-th/9812074] [INSPIRE].

[50] I.A. Bandos, J.A. de Azcarraga and D.P. Sorokin, On D = 11 supertwistors, superparticle quantization and a hidden $\mathrm{SO}(16)$ symmetry of supergravity, hep-th/0612252 [INSPIRE].

[51] I.A. Bandos, Spinor moving frame, M0-brane covariant BRST quantization and intrinsic complexity of the pure spinor approach, Phys. Lett. B 659 (2008) 388 [arXiv:0707.2336] [INSPIRE].

[52] I.A. Bandos, $D=11$ massless superparticle covariant quantization, pure spinor BRST charge and hidden symmetries, Nucl. Phys. B 796 (2008) 360 [arXiv:0710.4342] [InSPIRE].

[53] W. Siegel, Hidden Local Supersymmetry in the Supersymmetric Particle Action, Phys. Lett. B 128 (1983) 397 [INSPIRE].

[54] J.A. de Azcarraga and J. Lukierski, Supersymmetric Particles with Internal Symmetries and Central Charges, Phys. Lett. B 113 (1982) 170 [INSPIRE].

[55] J.A. de Azcarraga and J. Lukierski, Supersymmetric Particles in $N=2$ Superspace: Phase Space Variables and Hamiltonian Dynamics, Phys. Rev. D 28 (1983) 1337 [InSPIRE].

[56] E. Bergshoeff and P.K. Townsend, Super D-branes, Nucl. Phys. B 490 (1997) 145 [hep-th/9611173] [INSPIRE].

[57] N. Boulanger and P. Sundell, An action principle for Vasiliev's four-dimensional higher-spin gravity, J. Phys. A 44 (2011) 495402 [arXiv: 1102.2219] [INSPIRE].

[58] N. Boulanger, N. Colombo and P. Sundell, A minimal BV action for Vasiliev's four-dimensional higher spin gravity, JHEP 10 (2012) 043 [arXiv:1205.3339] [INSPIRE].

[59] M.A. Vasiliev, Equations of Motion of Interacting Massless Fields of All Spins as a Free Differential Algebra, Phys. Lett. B 209 (1988) 491 [INSPIRE].

[60] M.A. Vasiliev, Consistent Equations for Interacting Massless Fields of All Spins in the First Order in Curvatures, Annals Phys. 190 (1989) 59 [INSPIRE].

[61] M.A. Vasiliev, Consistent equation for interacting gauge fields of all spins in (3+1)-dimensions, Phys. Lett. B 243 (1990) 378 [INSPIRE].

[62] C. Fronsdal, Massless Fields with Integer Spin, Phys. Rev. D 18 (1978) 3624 [InSPIRE].

[63] N. Berkovits, Super Poincaré covariant quantization of the superstring, JHEP 04 (2000) 018 [hep-th/0001035] [INSPIRE].

[64] N. Berkovits, Pure spinor formalism as an $N=2$ topological string, JHEP 10 (2005) 089 [hep-th/0509120] [INSPIRE].

[65] N. Berkovits and N. Nekrasov, Multiloop superstring amplitudes from non-minimal pure spinor formalism, JHEP 12 (2006) 029 [hep-th/0609012] [INSPIRE]. 
[66] N. Berkovits and E. Witten, Supersymmetry Breaking Effects using the Pure Spinor Formalism of the Superstring, JHEP 06 (2014) 127 [arXiv: 1404.5346] [INSPIRE].

[67] I. Oda and M. Tonin, Y-formalism and $b$ ghost in the non-minimal pure spinor formalism of superstrings, Nucl. Phys. B 779 (2007) 63 [arXiv:0704.1219] [INSPIRE].

[68] A. Ferber, Supertwistors and Conformal Supersymmetry, Nucl. Phys. B 132 (1978) 55 [INSPIRE].

[69] T. Shirafuji, Lagrangian Mechanics of Massless Particles With Spin, Prog. Theor. Phys. 70 (1983) 18 [INSPIRE].

[70] E. Newman and R. Penrose, An approach to gravitational radiation by a method of spin coefficients, J. Math. Phys. 3 (1962) 566 [InSPIRE].

[71] R. Penrose and W. Rindler, Spinors And Space-time: Volume 1. Two Spinor Calculus And Relativistic Fields, Cambridge Monographs On Mathematical Physics, Cambridge University Press, Cambridge, U.K. (1984), pg. 458.

[72] R. Penrose and W. Rindler, Spinors And Space-time: Volume 2. Spinor And Twistor Methods In Space-time Geometry, Cambridge Monographs On Mathematical Physics, Cambridge University Press, Cambridge, U.K. (1986), pg. 501.

[73] D.V. Uvarov, (Super)twistors and (super)strings, Class. Quant. Grav. 23 (2006) 2711 [hep-th/0601149] [INSPIRE].

[74] D.V. Uvarov, Supertwistor formulation for higher dimensional superstrings, Class. Quant. Grav. 24 (2007) 5383 [hep-th/0703051] [INSPIRE].

[75] D.V. Uvarov, Canonical description of $D=10$ superstring formulated in supertwistor space, J. Phys. A 42 (2009) 115204 [arXiv:0804.0908] [inSPIRE].

[76] S. Fedoruk and J. Lukierski, Twistorial versus space-time formulations: Unification of various string models, Phys. Rev. D 75 (2007) 026004 [hep-th/0606245] [INSPIRE].

[77] S. Fedoruk and J. Lukierski, Purely twistorial string with canonical twistor field quantization, Phys. Rev. D 79 (2009) 066006 [arXiv:0811.3353] [INSPIRE].

[78] I.A. Bandos and C. Meliveo, Covariant action and equations of motion for the eleven dimensional multiple M0-brane system, Phys. Rev. D 87 (2013) 126011 [arXiv:1304.0382] [INSPIRE].

[79] N. Berkovits and E. Witten, Conformal supergravity in twistor-string theory, JHEP 08 (2004) 009 [hep-th/0406051] [INSPIRE].

[80] M. Abou-Zeid, C.M. Hull and L.J. Mason, Einstein Supergravity and New Twistor String Theories, Commun. Math. Phys. 282 (2008) 519 [hep-th/0606272] [INSPIRE].

[81] J.W. van Holten and A. Van Proeyen, $N=1$ Supersymmetry Algebras in $D=2, D=3$, $D=4$ MOD-8, J. Phys. A 15 (1982) 3763 [InSPIRE].

[82] T. Curtright, Are There Any Superstrings in Eleven-dimensions?, Phys. Rev. Lett. 60 (1988) 393 [Erratum ibid. 60 (1988) 1990] [INSPIRE].

[83] I.A. Bandos and J. Lukierski, Tensorial central charges and new superparticle models with fundamental spinor coordinates, Mod. Phys. Lett. A 14 (1999) 1257 [hep-th/9811022] [INSPIRE]. 
[84] C. Chryssomalakos, J.A. de Azcarraga, J.M. Izquierdo and J.C. Perez Bueno, The geometry of branes and extended superspaces, Nucl. Phys. B 567 (2000) 293 [hep-th/9904137] [INSPIRE].

[85] I.A. Bandos, J.A. de Azcarraga, J.M. Izquierdo and J. Lukierski, BPS states in M-theory and twistorial constituents, Phys. Rev. Lett. 86 (2001) 4451 [hep-th/0101113] [INSPIRE].

[86] I.A. Bandos, J.A. de Azcarraga, M. Picón and O. Varela, Supersymmetric string model with 30 kappa symmetries and extended supersapce and 30-32 BPS states, Phys. Rev. D 69 (2004) 085007 [hep-th/0307106] [InSPIRE].

[87] R. D'Auria and P. Fré, Geometric Supergravity in D = 11 and Its Hidden Supergroup, Nucl. Phys. B 201 (1982) 101 [Erratum ibid. B 206 (1982) 496] [InSPIRE].

[88] I.A. Bandos, J.A. de Azcarraga, J.M. Izquierdo, M. Picón and O. Varela, On the underlying gauge group structure of D=11 supergravity, Phys. Lett. B 596 (2004) 145 [hep-th/0406020] [INSPIRE].

[89] I.A. Bandos, J.A. de Azcarraga, M. Picón and O. Varela, On the formulation of $D=11$ supergravity and the composite nature of its three-form gauge field, Annals Phys. 317 (2005) 238 [hep-th/0409100] [INSPIRE].

[90] I.A. Bandos, J.A. de Azcarraga, J.M. Izquierdo, M. Picón and O. Varela, On BPS preons, generalized holonomies and $D=11$ supergravities, Phys. Rev. D 69 (2004) 105010 [hep-th/0312266] [INSPIRE].

[91] N.E.J. Bjerrum-Bohr, P.H. Damgaard, P. Tourkine and P. Vanhove, Scattering Equations and String Theory Amplitudes, arXiv:1403.4553 [INSPIRE].

[92] Y. Geyer, A.E. Lipstein and L.J. Mason, Ambitwistor strings in 4-dimensions, Phys. Rev. Lett. 113 (2014) 081602 [arXiv:1404.6219] [INSPIRE]. 